\title{
Two-beam light with simultaneous anticorrelations in photon-number fluctuations and sub-Poissonian statistics
}

\author{
Jan Peřina Jr.,, , $⿴ 囗 十$ Václav Michálek, ${ }^{1}$ Radek Machulka, ${ }^{2}$ and Ondřej Haderka ${ }^{2}$ \\ ${ }^{1}$ Joint Laboratory of Optics of Palacky University and Institute of Physics of the Czech Academy of Sciences, \\ Faculty of Science, Palacký University, 17. listopadu 12, 77146 Olomouc, Czech Republic \\ ${ }^{2}$ Institute of Physics of the Czech Academy of Sciences, \\ Joint Laboratory of Optics of Palacký University and Institute of Physics of CAS, \\ 17. listopadu 50a, 772 07 Olomouc, Czech Republic
}

\begin{abstract}
Two twin beams with a shared signal beam and separated idler beams are used together with the photon-number-resolving postselection in the signal beam to arrive at two coupled beams with anticorrelations in photon-number fluctuations. Moreover, the beams exhibit the sub-Poissonian photon-number statistics in their marginal distributions under suitable conditions. The postselected fields with the increasing mean photon numbers are reconstructed from the experimental photocount histograms by the maximum likelihood approach. Also a suitable Gaussian fit of both original twin beams and simulation of the postselection process are applied to arrive at the corresponding photon-number distributions. Their nonclassical properties are analyzed by suitable nonclassicality criteria and quantified by the corresponding nonclassicality depths. Determining the appropriate quasi-distributions of integrated intensities with negative values, the performance of different nonclassicality criteria is judged. Properties of the postselected fields reached both by the used and ideal photon-number-resolved detectors are mutually compared.
\end{abstract}

\section{INTRODUCTION}

Twin beams (TWBs) generated in spontaneous parametric down-conversion [1] are endowed with highly nonclassical properties [2] observed in different degrees of freedom. Their entanglement occurring in the polarization degrees of freedom has been exploited to test the quantum mechanics via the violation of the Bell inequalities [3, 4] or to teleport the polarization state of a photon [5]. Tight spatial correlations of the photons in a TWB lie in the heart of quantum imaging [6]. On the other hand perfect correlations in photon numbers of the signal and idler beams, that constitute a TWB, 7 11] gave rise to the method of absolute detector calibration [12 15].

Also, a very efficient method for sub-Poissonian light generation by photon-number-resolved postselection (in cw regime: [16 18], in pulsed regime: [9, 19 22]) is based upon TWBs. Such states represent a generalization of (heralded) single-photon Fock states [23 27] to more intense fields described in the Hilbert spaces of larger dimensions. Such fields then allow, among others, to increase the capacity of communication channels [28]. The highly-nonclassical single-photon Fock states are a workhorse of the broad area of quantum-information processing 29] based on the discrete variables. They also find their application in sub-shot-noise imaging [30-34].

The used postselection process represents a critical step in the preparation of highly-nonclassical states as it degaussifies the original Gaussian TWB. This makes the postselection method very prospective for the generation of more complex quantum states potentially needed in future quantum-information protocols that will go be-

\footnotetext{
* jan.perina.jr@upol.cz
}

yond the single-photon Fock states. Also the application of such states in quantum metrology [31, 35 37] is expected. We note that the generation of photon-numbersubtracted states [38 40] represents a special variant of the postselection with photon-number-resolving detectors that allows to generate various kinds of nonclassical states, even from TWBs [41, 42].

To put our considerations about the states with different photon numbers and photon-number correlations into the general context, we remind the reader that, according to the second-quantization of electromagnetic fields in the quantum mechanics 2], any state of an optical field can be decomposed into the base vectors of the general Hilbert space spanned over the spatio-spectral, polarization and amplitude (field quantization) degrees of freedom. Whereas the majority of the experiments with individual photon pairs are realized by manipulating the states in spatio-spectral and/or polarization degrees of freedom while keeping the state in the amplitude degree of freedom fixed, we use the opposite configuration: We do not consider the spatio-spectral and polarization degrees of freedom explicitly (we trace them out) and we modify and transform the states only in the Hilbert space belonging to the amplitude degree of freedom, i.e. the space spanned by the Fock states of different photon numbers.

Here, we further develop and utilize the method of photon-number-resolved postselection from TWBs to open the door for the generation of a new class of quantum states exhibiting anticorrelations in photon-number fluctuations and marginal sub-Poissonian statistics. To arrive at such states we consider two TWBs with their signal beams detected together and postselection on the shared signal beam by observing a given number of signal photons $n_{s}$. The remaining two idler beams are left in a state that exhibits strong anticorrelations in their 
photon-number fluctuations. This is in striking contrast with the usual TWBs exhibiting perfect correlations in photon numbers as well as their fluctuations. Moreover, whereas the marginal photon-number statistics of TWBs are super-Poissonian, the obtained states exhibit the marginal sub-Poissonian photon-number statistics [39]. These states are prospective for metrology: They allow to measure two-photon absorption cross-sections with the precision below the shot-noise limit, in close analogy with the sub-shot-noise measurement of single-photon absorption cross-section performed with a sub-Poissonian light source [30, 33, 34].

We note that there exists an analogy between the anticorrelations in the photon-number fluctuations of the analyzed fields and the spatial and temporal behavior of correlations between the signal and idler photons from a common photon pair. Thought both photons from a photon pair usually show strong temporal correlations [43], these correlations can be transformed into temporal anticorrelations [44]. Similarly, whereas the signal and idler photons of usual TWBs are bunched inside their correlated areas, there also exist the TWBs exhibiting spatial antibunching of the signal and idler photons [45, 46].

The suggested scheme resembles that of the entanglement swapping suggested first for the states of two entangled photon pairs [47, 48] originating in parametric down-conversion and later also applied to swap the entanglement to the state of particles and their collective modes [49, 50]. However, sensitivity of the detected overall signal beam to the relative phase of the constituting signal beams would be needed to observe the transfer of entanglement from the original TWBs to the postselected idler beams. As the used TWBs are multi-mode, they are not suitable for the entanglement transfer. Instead, in the performed experiment, the postselection induces classical anticorrelations in photon-number fluctuations.

To demonstrate the essence of our approach, we restrict for a moment our attention to the states describing single-mode idler beams and consider an ideal detector with $n_{\mathrm{s}}$ detected signal photons (photocounts). We model the experimental multi-mode idler beams by an incoherent superposition of the Fock states whose statistical operator $\hat{\varrho}_{\mathrm{ii}}$ is written as

$$
\hat{\varrho}_{\mathrm{ii}}=\sum_{\mathrm{i}_{1}=0}^{n_{\mathrm{s}}}\left|\alpha_{\mathrm{i}_{1}}\right|^{2}\left|n_{\mathrm{i}_{1}}\right\rangle{\mathrm{i}_{1} \mathrm{i}_{1}}_{\mathrm{i}_{1}}\left\langle n_{\mathrm{i}_{1}}|| n_{\mathrm{s}}-n_{\mathrm{i}_{1}}\right\rangle_{\mathrm{i}_{2} \mathrm{i}_{2}}\left\langle n_{\mathrm{s}}-n_{\mathrm{i}_{1}}\right| .
$$

In Eq. (11), a Fock state $\left|n_{\mathrm{i}}\right\rangle_{\mathrm{i}}$ has $n_{\mathrm{i}}$ photons in beam $\mathrm{i}$ and $\alpha_{\mathrm{i}}$ are complex coefficients. Anticorrelations in photonnumber fluctuations $\Delta n_{\mathrm{i}} \equiv n_{\mathrm{i}}-\left\langle n_{\mathrm{i}}\right\rangle$ represent the most striking feature of the state $\hat{\varrho}_{\mathrm{ii}}$. Detailed analysis reveals that even the marginal idler-beam distributions of the analyzed states are sub-Poissonian under suitable conditions. To understand this, let us consider for a moment the experiment in which we independently detect the numbers of signal photons in both signal beams. For the fixed detected signal photon numbers, both postselected idler beams have apparently sub-Poissonian statistics.
The summation of two signal photon numbers keeping their sum fixed, as described in Eq. (11), blurs the original sub-Poissonian statistics but it also increases the success probability of the postselection process. For TWBs with greater photon numbers and corresponding signal postselecting photon numbers [see Fig. 3(b) below], the blurring of the idler-beams photon statistics is weak, but the success probability increases considerably. Such states are then suitable for monitoring two-photon absorption processes or making two-photon excitations of electronic systems.

The paper is organized as follows. The performed experiment and analysis of the experimental data are described in Sec. II. Sec. III is devoted to the analysis of the fields generated by postselection with the real detector. The properties of the fields obtained by postselection with an ideal detector are discussed in Sec. IV. Detailed analysis of nonclassical properties of typical postselected fields is contained in Sec. V. Sec. VI gives the conclusions. In Appendix A, a method for fitting the experimental data with a suitable multi-mode Gaussian field is presented. Iteration formulas for the maximum-likelihood reconstruction are given in Appendix B. Nonclassicality identifiers are introduced in Appendix C. The formula for reconstructing quasi-distributions of integrated intensities is given in Appendix D.

\section{EXPERIMENTAL SETUP, RECONSTRUCTION AND NONCLASSICALITY ANALYSIS}

To analyze the performed experiment, we consider two multi-mode and noisy TWBs whose common mixed state is characterized by a 3D photon-number distribution $p\left(n_{\mathrm{s}}, n_{\mathrm{i}_{1}}, n_{\mathrm{i}_{2}}\right)$ that gives the probability of simultaneous presence of $n_{\mathrm{s}}$ photons in the signal beam, $n_{\mathrm{i}_{1}}$ photons in the first idler beam and $n_{\mathrm{i}_{2}}$ photons in the second idler beam (for specific photon-number distributions, see Appendix A). Characterizing a photon-number-resolving detector (PNRD) in the signal beam by its detection matrix $T_{\mathrm{s}}\left(c_{\mathrm{s}}, n_{\mathrm{s}}\right)$, that gives the probability of detecting $c_{\mathrm{s}}$ photocounts out of $n_{\mathrm{s}}$ impinging photons (for details, see Appendix A), 2D photon-number distribution $p_{\mathrm{ii}}\left(n_{\mathrm{i}_{1}}, n_{\mathrm{i}_{2}} ; c_{\mathrm{s}}\right)$ of a common state of the idler beams emerging after detecting $c_{\mathrm{s}}$ signal photocounts is written as [51]:

$$
p_{i i}\left(n_{\mathrm{i}_{1}}, n_{\mathrm{i}_{2}} ; c_{\mathrm{s}}\right)=\sum_{n_{\mathrm{s}}=0}^{\infty} T_{\mathrm{s}}\left(c_{\mathrm{s}}, n_{\mathrm{s}}\right) p\left(n_{\mathrm{s}}, n_{\mathrm{i}_{1}}, n_{\mathrm{i}_{2}}\right) .
$$

In the experiment, the postselected fields are monitored by two additional PNRDs that give rise, together with the PNRD in the signal beam, to the $3 \mathrm{D}$ experimental photocount histogram $f\left(c_{\mathrm{s}}, c_{\mathrm{i}_{1}}, c_{\mathrm{i}_{2}}\right)$ that contains all information about the prepared and analyzed fields. In the model, this histogram $f$, as a function of the photocount numbers $c_{\mathrm{s}}, c_{\mathrm{i}_{1}}$ and $c_{\mathrm{i}_{2}}$ registered by three used PNRDs, 
is determined along the formula

$$
\begin{aligned}
f\left(c_{\mathrm{s}}, c_{\mathrm{i}_{1}}, c_{\mathrm{i}_{2}}\right)= & \sum_{n_{\mathrm{s}}=0}^{\infty} T_{\mathrm{s}}\left(c_{\mathrm{s}}, n_{\mathrm{s}}\right) \sum_{n_{\mathrm{i}_{1}}=0}^{\infty} T_{\mathrm{i}_{1}}\left(c_{\mathrm{i}_{1}}, n_{\mathrm{i}_{1}}\right) \\
& \times \sum_{n_{\mathrm{i}_{2}}=0}^{\infty} T_{\mathrm{i}_{2}}\left(c_{\mathrm{i}_{2}}, n_{\mathrm{i}_{2}}\right) p\left(n_{\mathrm{s}}, n_{\mathrm{i}_{1}}, n_{\mathrm{i}_{2}}\right)
\end{aligned}
$$

in which the detection matrix $T_{\mathrm{i}_{1}}\left(T_{\mathrm{i}_{2}}\right)$ belongs to the PNRD placed in the first (second) idler beam.

The reconstruction methods allow us to reveal both the conditional 2D photon-number distributions $p_{\mathrm{ii}}$ in Eq. (3) as well as the original 3D photon-number distribution $p$. Both a physically-motivated method that provides a suitable Gaussian fit of the original two TWBs (see Appendix A) and a method exploiting the maximum-likelihood approach (see Appendix B) were applied to reconstruct the experimental photocount histogram $f\left(c_{\mathrm{s}}, c_{\mathrm{i}_{1}}, c_{\mathrm{i}_{2}}\right)$ as well as the conditional photocount histograms $f_{\mathrm{ii}}\left(c_{\mathrm{i}_{1}}, c_{\mathrm{i}_{2}} ; c_{\mathrm{S}}\right)$ characterizing the conditional $2 \mathrm{D}$ photon-number distributions $p_{\mathrm{ii}}\left(n_{\mathrm{i}_{1}}, n_{\mathrm{i}_{2}} ; c_{\mathrm{s}}\right)$.

The analyzed states were prepared in the lab in the experiment whose scheme is shown in Fig. 11 Two TWBs were generated independently in type-I spontaneous parametric down-conversion in two optically contacted 1-mm-long $\beta$-barium-borate composite crystals $\left(\mathrm{BaB}_{2} \mathrm{O}_{4}, \mathrm{BBO}\right)$ cut for a slightly non-collinear geometry. Whereas the first crystal gave the signal and idler beams with horizontal polarizations, the second crystal emitted the signal and idler beams with vertical polarizations, as a consequence of its rotation by 90 degrees along the pump-beam propagating direction with respect to the first crystal. Parametric down-conversion was pumped by pulses originating in the third harmonic $(280 \mathrm{~nm})$ of a femtosecond cavity-dumped Ti:sapphire laser (pulse duration $180 \mathrm{fs}$ at the central wavelength of $840 \mathrm{~nm}$, repetition rate $50 \mathrm{kHz}$, pulse energy $20 \mathrm{~nJ}$ at the output of the third harmonic generator). The polarization of the pump was then rotated by a half-wave plate to balance the mutually orthogonal contributions from both crystals. The idler beams of two TWBs that differ by their polarizations were spatially separated by a calcite beam displacer. The signal, two idler and external noise beams were detected in four different detection regions (in the form of strips) on the photocathode of an iCCD camera Andor DH345-18U-63 [see the rightmost image in Fig. 1(b)]. The signal beams emitted from different crystals spatially overlapped at the photocathode and so they were detected in a common detection region. The camera set for 7 ns-long detection window was driven by the synchronization electronic pulses from the laser and it operated roughly at $14 \mathrm{~Hz}$ frame rate. The photons of all four beams impinging on the camera were filtered by a 14-nm-wide bandpass interference filter with the central wavelength at $560 \mathrm{~nm}$. The pump intensity, and thus also the TWBs intensity, was actively stabilized by means of a motorized half-wave plate followed by a polarizer and a detector that monitored the actual pump intensity.
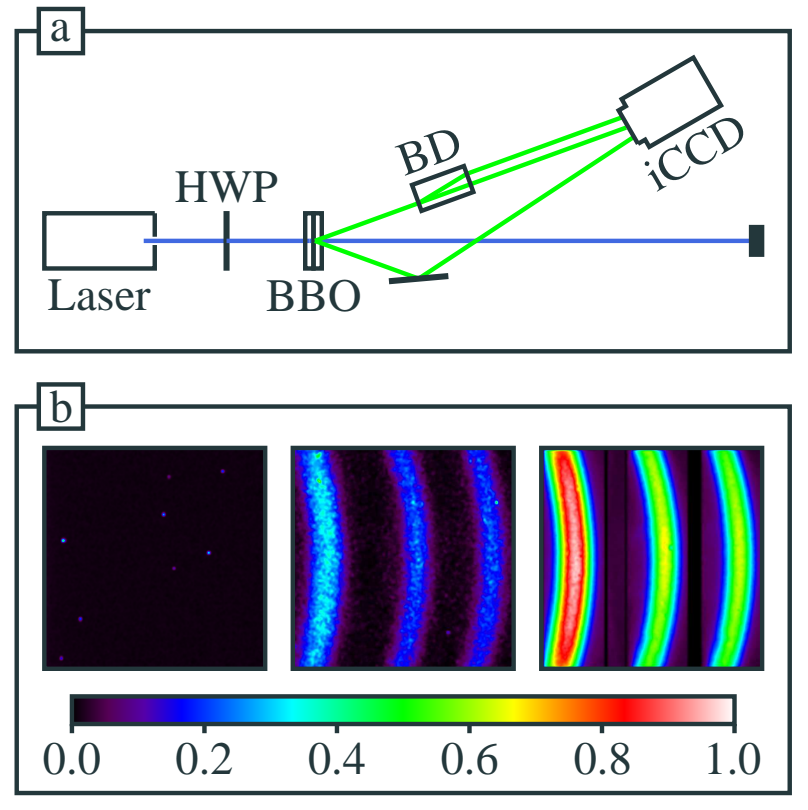

FIG. 1. (a) Scheme of the experimental setup: Laser: frequency-tripled pump laser with power stabilizer; HWP: half-wave plate; BBO: two thin optically contacted BBO crystals; BD: polarizing beam displacer; iCCD: intensified CCD camera. (b) Images acquired by the detector, in turn: typical single-shot image, accumulated image from multiple singleshot exposures forming one signal (left) and two idler (right) intense strips, and cumulative frame formed by individual detection events identified by signal processing within regions defined by one signal, one narrow noise (formed solely by the dark, ambient and readout noise) and two idler beams.

The Gaussian reconstruction applied to the experimental photocount histogram $f\left(c_{\mathrm{s}}, c_{\mathrm{i}_{1}}, c_{\mathrm{i}_{2}}\right)$ obtained after $1.2 \times 10^{6}$ measurement repetitions provided the following parameters for the optical fields beyond the nonlinear crystals: The overall field was composed of two ideal TWBs with $6.15 \pm 0.05$ and $5.95 \pm 0.05$ mean photon pairs $\left(B_{\mathrm{p}_{1}}=0.106 \pm 0.001, B_{\mathrm{p}_{2}}=0.117 \pm 0.001, M_{\mathrm{p}_{1}}=58 \pm 1\right.$, $\left.M_{\mathrm{p}_{2}}=51 \pm 1\right)$ and three noise fields with $0.11 \pm 0.02$, $0.07 \pm 0.01$ and $0.02 \pm 0.01$ mean noise photons $\left(B_{\mathrm{s}}=\right.$ $10 \pm 1, B_{\mathrm{i}_{1}}=10 \pm 1, B_{\mathrm{i}_{2}}=39 \pm 4, M_{\mathrm{s}}=0.011 \pm 0.001$, $\left.M_{\mathrm{i}_{1}}=0.007 \pm 0.001, M_{\mathrm{i}_{2}}=0.0005 \pm 0.0001\right) ; M_{j}$ stands for the number of modes in beam $j$ having $B_{j}$ mean photons (photon pairs) per mode (see Appendix A for more details). The signal field was detected with detection efficiency $\eta_{\mathrm{s}}=22.0 \pm 0.5 \%$, detection efficiency $\eta_{\mathrm{i}}=20.7 \pm 0.5 \%$ was assigned to both idler-field detection strips (lower than the signal one due to the presence of the beam displacer). Each detection strip was composed of $N_{\mathrm{s}}=N_{\mathrm{i}_{1}}=N_{\mathrm{i}_{2}}=4410$ macropixels (one macropixel emerged from $8 \times 8$ hardware binning at the CCD chip) and suffered from $d_{\mathrm{s}}=d_{\mathrm{i}_{1}}=d_{\mathrm{i}_{2}}=0.22 \pm 0.02$ mean noise counts per detection window.

The properties of the conditional states characterized by $2 \mathrm{D}$ photocount $\left[f_{\mathrm{ii}}\left(c_{\mathrm{i}_{1}}, c_{\mathrm{i}_{2}} ; c_{\mathrm{s}}\right)\right]$ and photon-number 
distributions $\left[p_{\mathrm{ii}}\left(n_{\mathrm{i}_{1}}, n_{\mathrm{i}_{2}} ; c_{\mathrm{s}}\right)\right]$ were quantified by the following parameters. Anticorrelation between the fluctuations $\Delta n(\Delta c)$ of the idler-fields photon (photocount) numbers was recognized by negative values of the covariance $C_{n, \Delta}$,

$$
C_{n, \Delta}=\frac{\left\langle\Delta n_{\mathrm{i}_{1}} \Delta n_{\mathrm{i}_{2}}\right\rangle}{\sqrt{\left\langle\left(\Delta n_{\mathrm{i}_{1}}\right)^{2}\right\rangle\left\langle\left(\Delta n_{\mathrm{i}_{2}}\right)^{2}\right\rangle}} .
$$

Nonclassical character of the conditional 2D idler fields is verified by the values of the modified noise-reductionparameter $R_{n,+}$ smaller than 1 ,

$$
R_{n,+}=\frac{\left\langle\left(\Delta\left(n_{\mathrm{i}_{1}}+n_{\mathrm{i}_{2}}\right)^{2}\right\rangle\right.}{\left\langle n_{\mathrm{i}_{1}}\right\rangle+\left\langle n_{\mathrm{i}_{2}}\right\rangle} .
$$

We have $R_{n,+}=1$ for two independent Poissonian fields in coherent states. Declinations of classical photonnumber distributions from the Poissonian ones as well as mutual photon-number correlations between the fields increase the values of the modified noise-reductionparameter $R_{n,+}>1$. On the other hand, the inequality $R_{n,+}<1$ is equivalent to the inequality for the moments of integrated intensities $\left\langle\left[\Delta\left(W_{\mathrm{i}_{1}}+W_{\mathrm{i}_{2}}\right)\right]^{2}\right\rangle \equiv$ $\int_{0}^{\infty} d W_{\mathrm{i}_{1}} \int_{0}^{\infty} d W_{\mathrm{i}_{2}}\left[\Delta\left(W_{\mathrm{i}_{1}}+W_{\mathrm{i}_{2}}\right)\right]^{2} P_{\mathcal{N}}\left(W_{\mathrm{i}_{1}}, W_{\mathrm{i}_{2}}\right)<0$. Its fulfillment requires the quasi-distribution $P_{\mathcal{N}}\left(W_{\mathrm{i}_{1}}, W_{\mathrm{i}_{2}}\right)$ of integrated intensities with negative values which implies the fields' nonclassicality. We note that the integrated intensities $W$ and their moments occur in the description of optical fields in relation to their detection as the fields detectors are sensitive to the normally-ordered photon-number moments that are referred to as the moments of integrated intensity [for the relation between both types of moments, see Eq. (A6) in Appendix A]. We have $R_{n,+}=0$ for the state in Eq. (11). Thus, this state in nonclassical. On the other hand, it is not entangled as it contains only classical anticorrelations in photon-number fluctuations.

Also the marginal idler fields may exhibit the nonclassical sub-Poissonian statistics observed when the values of the Fano factors $F_{n, \mathrm{i}_{j}}, j=1,2$,

$$
F_{n, \mathrm{i}_{j}}=\frac{\left\langle\left(\Delta n_{\mathrm{i}_{j}}\right)^{2}\right\rangle}{\left\langle\Delta n_{\mathrm{i}_{j}}\right\rangle}
$$

are smaller than 1 .

The nonclassicality of conditional 2D idler fields may be identified both using the nonclassicality criteria (NCCa) written in terms of the intensity moments and probabilities of photon-number (photocount) distributions. The NCCa using the intensity moments $C_{W}$ and $M_{W}$,

$$
\begin{aligned}
C_{W} \equiv & \left\langle W_{\mathrm{i}_{1}}^{2} W_{\mathrm{i}_{2}}^{2}\right\rangle-\left\langle W_{\mathrm{i}_{1}} W_{\mathrm{i}_{2}}\right\rangle^{2}<0, \\
M_{W} \equiv & \left\langle W_{\mathrm{i}_{1}}^{2}\right\rangle\left\langle W_{\mathrm{i}_{2}}^{2}\right\rangle+2\left\langle W_{\mathrm{i}_{1}} W_{\mathrm{i}_{2}}\right\rangle\left\langle W_{\mathrm{i}_{1}}\right\rangle\left\langle W_{\mathrm{i}_{2}}\right\rangle-\left\langle W_{\mathrm{i}_{1}} W_{\mathrm{i}_{2}}\right\rangle^{2} \\
& -\left\langle W_{\mathrm{i}_{1}}^{2}\right\rangle\left\langle W_{\mathrm{i}_{2}}\right\rangle^{2}-\left\langle W_{\mathrm{i}_{1}}\right\rangle^{2}\left\langle W_{\mathrm{i}_{2}}^{2}\right\rangle<0 .
\end{aligned}
$$

derived from the Cauchy-Schwarz inequality and the matrix approach [52], respectively, have been found the most powerful for the analyzed states. They belong to the groups of the NCCa discussed in Appendix C $\left[C_{W}=C_{(1,1)}^{(2,0)}, M_{W}=M_{(0,0),(1,0),(0,1)}\right]$. Their probability variants are then used to identify the location of nonclassicality across the probability distributions.

Sub-Poissonian character of the marginal idler fields makes the following hybrid NCC $L$ [53] very efficient in revealing the nonclassicality:

$$
L_{W p}\left(n_{\mathrm{i}_{1}}\right) \equiv\left\langle W_{i_{2}}^{3}\right\rangle_{n_{\mathrm{i}_{1}}}\left\langle W_{i_{2}}\right\rangle_{n_{\mathrm{i}_{1}}}-\left\langle W_{i_{2}}^{2}\right\rangle_{n_{\mathrm{i}_{1}}}^{2}<0 .
$$

In Eq. (9), averaging \langle\rangle$_{n_{\mathrm{i}_{1}}}$ is performed in the variable $n_{\mathrm{i}_{2}}$ with the photon-number distribution $p\left(n_{\mathrm{i}_{1}}, n_{\mathrm{i}_{2}}\right)$ in which $n_{\mathrm{i}_{1}}$ is kept fixed. This means that the intensity moments are determined in one variable whereas the probabilities are used in the other to reveal the nonclassicality.

When applying the concept of the Lee nonclassicality depth (NCD) 54 the NCCa also provide quantification of the nonclassicality. The NCD $\tau$ is derived from the value $s_{\text {th }}$ of the ordering parameter at which the used NCC loses its ability to reveal the nonclassicality of the analyzed field [52]:

$$
\tau=\left(1-s_{\mathrm{th}}\right) / 2 .
$$

To determine the threshold values $s_{\mathrm{th}}$, transformations of the photon-number distributions as well as the intensity moments between different field's orderings are needed 55 57].

\section{NONCLASSICAL LIGHT GENERATED BY POSTSELECTION WITH THE REAL DETECTOR}

First, we analyze the experimental 2D photocount histograms $f_{\mathrm{ii}}\left(c_{\mathrm{i}_{1}}, c_{\mathrm{i}_{2}} ; c_{\mathrm{s}}\right)$ and the corresponding reconstructed photon-number distributions reached by the maximum-likelihood approach $\left[p_{\mathrm{ii}}^{M L}\left(n_{\mathrm{i}_{1}}, n_{\mathrm{i}_{2}} ; c_{\mathrm{s}}\right)\right.$, see Appendix B] and the suitable Gaussian fit $\left[p_{\mathrm{ii}}^{G}\left(n_{\mathrm{i}_{1}}, n_{\mathrm{i}_{2}} ; c_{\mathrm{s}}\right)\right.$, see Appendix A] from the point of view of the marginal idler-fields mean photocount $\left[\left\langle c_{\mathrm{i}_{j}}\right\rangle, j=1,2\right]$ and photon $\left[\left\langle n_{\mathrm{i}_{j}}\right\rangle\right]$ numbers and the Fano factors $\left[F_{\mathrm{i}_{j}}\right]$ that quantify the spread of photocount and photon-number fluctuations. Both marginal idler fields behave similarly. The mean photocount $\left[\left\langle c_{\mathrm{i}_{1}}\right\rangle\right]$ and photon $\left[\left\langle n_{\mathrm{i}_{1}}\right\rangle\right]$ numbers of the first idler field increase with the increasing postselected signal photocount number $c_{\mathrm{s}}$ in the analyzed range $c_{\mathrm{s}} \leq 10$, as shown in Fig. 2(a). On the other hand, the relative fluctuations in photocount and photon numbers as quantified by the Fano factors $F_{c, \mathrm{i}_{1}}$ and $F_{n, \mathrm{i}_{1}}$ in Fig. 2(b) decrease with the increasing $c_{\mathrm{s}}$ up to $c_{\mathrm{s}}=7$ and then they increase. This is a consequence of the postselection mechanism between the signal and the first idler field that suffers from non-unit detection efficiency $\eta_{\mathrm{s}}$ and the noise signal photons together with the signaldetector dark counts. Whereas the detrimental role of non-unit detection efficiency $\eta_{\mathrm{s}}$ on the Fano factor $F$ decreases with the increasing signal photocount number $c_{\mathrm{s}}$, 


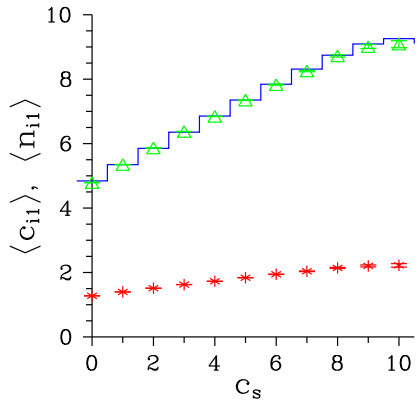

(a)

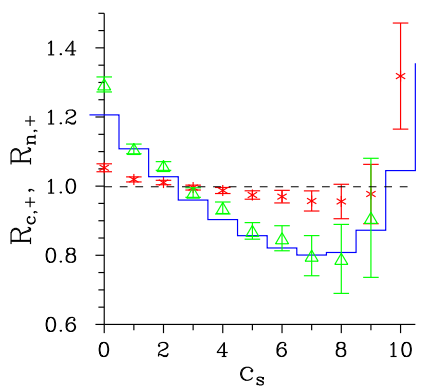

(c)

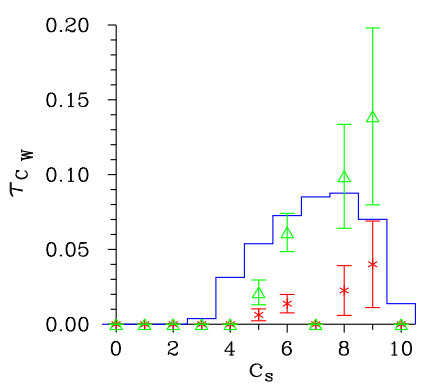

(e)

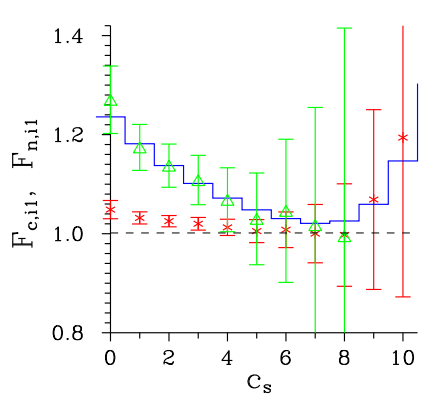

(b)

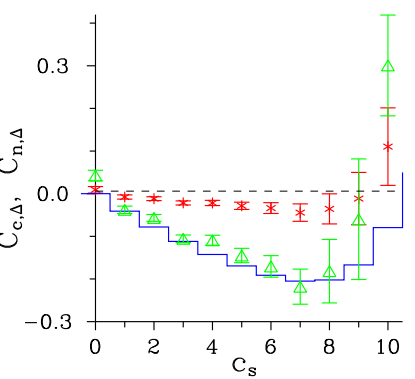

(d)

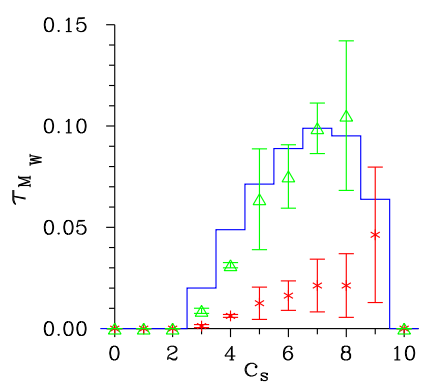

(f)
FIG. 2. (a) Mean number of photons $\left\langle n_{\mathrm{i}_{1}}\right\rangle$ (photocounts $\left\langle c_{\mathrm{i}_{1}}\right\rangle$ ) and (b) Fano factor $F_{n, \text { i }_{1}}\left(F_{c, \text { i }_{1}}\right)$ of the first idler field, (c) modified noise-reduction-parameter $R_{n,+}\left(R_{c,+}\right)$, (d) covariance $C_{n, \Delta}\left(C_{c, \Delta}\right)$, and nonclassicality depths (e) $\tau_{C_{W}}$ and (f) $\tau_{M_{W}}$ of the $2 \mathrm{D}$ idler fields observed after postselection as they depend on the signal-field postselecting photocount number $c_{\mathrm{s}}$. Isolated symbols are drawn for the experimental photocount histograms (red $*$ ) and fields reconstructed by 2D maximumlikelihood approach (green $\triangle$ ); solid blue curves originate in the 3D Gaussian model. The horizontal dashed lines indicate the borders of anticorrelation $\left(C_{\Delta}=0\right)$ and nonclassicality $\left(F_{\mathrm{i}_{1}}=1, R_{+}=1\right)$ regions.

the effect of the noise signal photons and dark-counts behaves in the opposed way [19]. Also the experimental errors of the Fano factor $F$ increase with the increasing $c_{\mathrm{S}}$ which is a consequence of the decreasing number of measurement repetitions associated with a given signal photocount number $c_{\mathrm{s}}$. Owing to the relatively low detection efficiency $\eta_{\mathrm{s}} \approx 20 \%$ and large relative portion of the noise in the signal field (around $1 / 2$ caused by the signal photons from the second TWB) the values of Fano factor $F$ remain in the classical region with $F \geq 1$.

However, when we analyze the performance of the postselection mechanism on the sum $c_{\mathrm{i}_{1}}+c_{\mathrm{i}_{2}}\left(n_{\mathrm{i}_{1}}+n_{\mathrm{i}_{2}}\right)$ of the first and the second idler photocount (photon) numbers, i.e. when the postselection mechanism works simultaneously and 'in-phase' on both TWBs, we get the reduction of fluctuations of the above sums below their classical border $\left(R_{+}<1\right)$ for $c_{\mathrm{s}} \in\langle 3,9\rangle$, as documented by the modified noise-reduction-parameters $R_{c,+}$ and $R_{n,+}$ plotted in Fig. 2(c). The smallest values of $R_{+}$indicating the strongest achieved suppression of the fluctuations are reached for the signal photocount numbers $c_{\mathrm{s}}=7,8$, in accordance with the behavior of the first and the second idler-field Fano factors $F_{\mathrm{i}_{1}}$ and $F_{\mathrm{i}_{2}}$. The suppression of fluctuations in the sum $c_{\mathrm{i}_{1}}+c_{\mathrm{i}_{2}}\left(n_{\mathrm{i}_{1}}+n_{\mathrm{i}_{2}}\right)$ of the idler-fields photocount (photon) numbers quantified by $R_{c,+}<1\left(R_{n,+}<1\right)$ gives rise to strong anticorrelations between the fluctuations of the first and the second photocount (photon) numbers $\Delta c_{\mathrm{i}_{1}}\left(\Delta n_{\mathrm{i}_{1}}\right)$ and $\Delta c_{\mathrm{i}_{2}}\left(\Delta n_{\mathrm{i}_{2}}\right)$. They are alternatively quantified by the covariances $C_{c, \Delta}$ and $C_{n, \Delta}$ drawn in Fig. 2(d).

Contrary to the case of TWBs, revealing the nonclassicality of the postselected 2D fields is much harder. Out of numerous NCCa written in intensity moments and successfully applied to TWBs in [56], only the NCC $C_{W}$ in Eq. (7) derived from the Cauchy-Schwarz inequality and the NCC $M_{W}$ in Eq. (8) originating in the matrix approach provided high and comparable values of the corresponding NCDs $\tau_{C_{W}}$ and $\tau_{M_{W}}$, as shown in Figs. 2(e,f). The comparison of graphs in Figs. $2(\mathrm{e})$ and 2 (f) drawn for the experimental 2D photocount histograms and photon-number distributions provided by $2 \mathrm{D}$ maximum-likelihood approach reveals the NCC $M_{W}$ as more stable and reliable because it identifies all the states postselected by detecting the signal photocount numbers $c_{\mathrm{s}} \in\langle 3,9\rangle$ as nonclassical, in accordance with the values of the modified noise-reductionparameter $R_{n,+}$ plotted in Fig. 2(c).

We note that the classical/nonclassical features identified in the experimental photocount histograms $f_{\text {ii }}$ are emphasized in the photon-number distributions $p_{\text {ii }}$ obtained by both reconstruction methods, as documented in Figs. 2(b-f).

\section{NONCLASSICAL LIGHT GENERATED BY POSTSELECTION WITH AN IDEAL DETECTOR}

Detection of the postselecting signal field with a better detection efficiency $\eta_{\mathrm{s}}$ opens the door for the observation of the postselected $2 \mathrm{D}$ idler fields with their most pronounced properties: anticorrelation in the idler-field photon-number fluctuations and sub-Poissonian statistics in the marginal idler fields. We demonstrate these properties by reconstructing the whole optical field as it occurs in front of all three used PNRDs, i.e. we also involve the signal-field postselecting detector in the reconstruction. We accomplish the reconstruction both by applying the 3D maximum-likelihood approach (see Appendix B) and a suitable 3D Gaussian fit (see Ap- 
pendix A) to the experimental photocount histogram $f\left(c_{\mathrm{S}}, c_{\mathrm{i}_{1}}, c_{\mathrm{i}_{2}}\right)$. Then, similarly as above, we analyze the $2 \mathrm{D}$ idler-fields photon-number distributions $p\left(n_{\mathrm{i}_{1}}, n_{\mathrm{i}_{2}} ; n_{\mathrm{s}}\right)$ conditioned by the presence of $n_{\mathrm{s}}$ photons in the signal field. This corresponds to the use of an ideal PNRD in the postselection mechanism.

The postselected idler fields behave similarly also in this case. The mean photon numbers $\left\langle n_{\mathrm{i}_{1}}\right\rangle^{\text {id }}$ of the first idler field increase roughly linearly with the postselecting signal photon number $n_{\mathrm{s}}$, and we have $\left\langle n_{\mathrm{i}_{1}}\right\rangle^{\text {id }} \approx n_{\mathrm{s}} / 2$ [see Fig. [3(a)]. Owing to the ideal detection efficiency $\eta_{\mathrm{s}}=1$ the Fano factors $F_{\mathrm{i}_{1}}$ attain nonclassical values $(F<1)$ for greater signal photon numbers $n_{\mathrm{s}}$. According to the graph in Fig. 3(b), the Fano factors $F^{\text {id }}$ smaller than 0.7 are reached for the signal photon numbers $n_{\mathrm{s}} \in\langle 4,20\rangle$. For the reconstructed 3D Gaussian field, sub-Poissonian character of the marginal idler fields is lost fast for even greater values of $n_{\mathrm{s}}$ as a consequence of the noise signal photons originating in the second TWB.

The sub-Poissonian Fano factors of the marginal idler fields reflect efficient functioning of the postselection mechanism that gives raise to low values of the modified noise-reduction-parameter $R_{n,+}^{\text {id }}$. According to Fig. 3(c) they attain the highly-nonclasical values around $0.2-$ 0.3 in the whole range $n_{\mathrm{s}} \in\langle 4,20\rangle$. Also the covariance $C_{n, \Delta}^{\mathrm{id}}$ of the idler-field photon-number fluctuations $\Delta n_{\mathrm{i}_{1}}$ and $\Delta n_{\mathrm{i}_{2}}$ plotted in Fig. B(d) attains the values around $-0.8--0.6$ in this range, which expresses the strong anticorrelation. Whereas the greatest values of the NCDs $\tau_{C_{W}}$ and $\tau_{M_{W}}$ reached by the real detector equal around 0.1 , the postselection by the ideal detector provides the much-greater values of up to around 0.4 , as documented in Figs. $3(\mathrm{e}, \mathrm{f})$. The comparison of graphs in Figs. 3(e) and 3(f) plotted for the photon-number distributions originating in the 3D maximum-likelihood approach reveals the NCC $M_{W}$ more stable than the NCC $C_{W}$ in identifying and quantifying the nonclassicality.

In the quantities plotted in Figs. 3(b-f) there occur little oscillations with the increasing period as the postselecting signal photon number $n_{\mathrm{s}}$ increases. They originate in the discrete photocount numbers $c_{\mathrm{s}}$ provided by the measurement. The 3D maximum-likelihood reconstruction has to correct for the detection efficiency $\eta_{\mathrm{s}} \approx 20 \%$ : The neighbor measurements for $c_{\mathrm{s}}$ and $c_{\mathrm{s}}+1$ postselecting photocounts have to be expanded into the interval of $n_{\mathrm{s}}$ postselecting photons from $\approx c_{\mathrm{s}} / \eta_{\mathrm{s}}$ to $\approx c_{\mathrm{s}} / \eta_{\mathrm{s}}+1 / \eta_{\mathrm{s}}$. Gradual stretching of the oscillation period $\approx 5$ is then caused by the presence of dark counts. The oscillations reflect the varying quality of the measurement for different postselecting photon numbers $n_{\mathrm{s}}$ : The measurements for the numbers $n_{\mathrm{s}}$ for which $\eta_{\mathrm{s}} n_{\mathrm{s}}$ are close to integers are of the best quality and allow to reconstruct the studied quantities in the best way. For the remaining numbers $n_{\mathrm{s}}$ the measurements are, roughly speaking, split between the neighbor photocount numbers $c_{\mathrm{s}}$ and so their quality as well as the quality of the reconstructed quantities are worse.

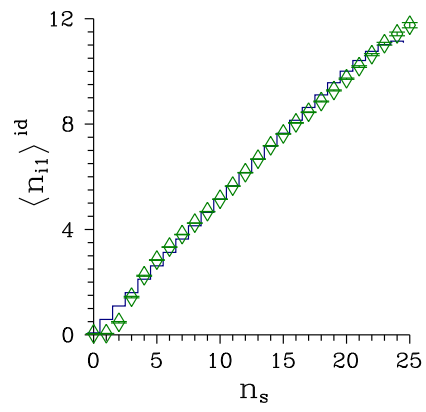

(a)

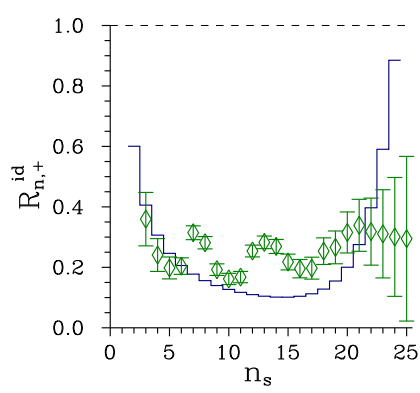

(c)

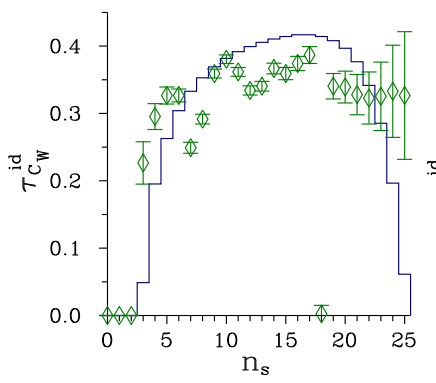

(e)

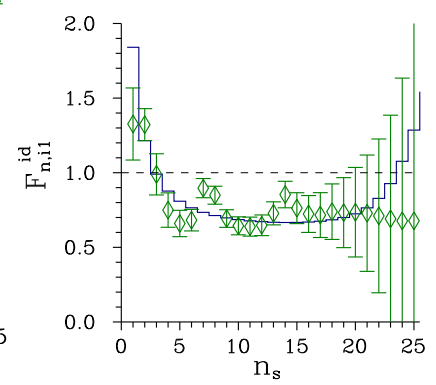

(b)

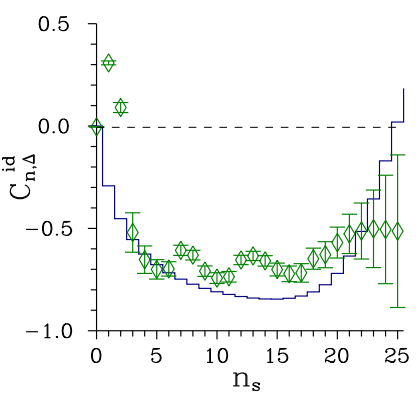

(d)

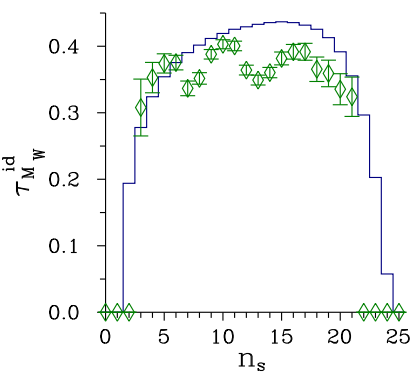

(f)
FIG. 3. (a) Mean number of photons $\left\langle n_{\mathrm{i}_{1}}\right\rangle^{\text {id }}$ and (b) Fano factor $F_{n, \mathrm{i}_{1}}^{\mathrm{id}}$ of the first idler field, (c) modified noise-reductionparameter $R_{n,+}^{\mathrm{id}}$, (d) covariance $C_{n, \Delta}^{\mathrm{id}}$, and nonclassicality depths (e) $\tau_{C_{W}}^{\mathrm{id}}$ and (f) $\tau_{M_{W}}^{\mathrm{id}}$ of the $2 \mathrm{D}$ idler fields reached by the ideal photon-number-resolving postselection as they depend on the signal-field postselecting photon number $n_{\mathrm{s}}$. Isolated symbols are drawn for the field reconstructed by $3 \mathrm{D}$ maximum-likelihood approach (dark green $\diamond)$; solid dark blue curves originate in the 3D Gaussian model. The horizontal dashed lines indicate the borders of anticorrelation $\left(C_{\Delta}^{\mathrm{id}}=0\right)$ and nonclassicality $\left(F_{\mathrm{i}_{1}}^{\mathrm{id}}=1, R_{+}^{\mathrm{id}}=1\right)$ regions.

\section{DETAILED ANALYSIS OF NONCLASSICAL PROPERTIES OF POSTSELECTED FIELDS}

Now we compare side-by-side the properties of two typical postselected states obtained by the real detector $\left(c_{\mathrm{s}}=5\right)$ and the ideal one $\left(n_{\mathrm{s}}=10\right)$. The state generated in the experimental setup by the real detector is a bit more intense, it contains on average around 7 photons in each idler field compared to around 5 photons in the idler fields of the state provided by the ideal detector. The correspoding 2D photon-number distri- 
butions $p_{i i}$ and $p_{i i}^{\text {id }}$ plotted in Figs. 4(a) and 4(b), respectively, clearly exhibit prolongation in the direction perpendicular to the line $n_{\mathrm{i}_{1}}=n_{\mathrm{i}_{2}}$. Whereas the covariance $C_{n, \Delta}$ of the idler-fields photon-number fluctuations $\Delta n_{\mathrm{i}_{1}}$ and $\Delta n_{\mathrm{i}_{2}}$ equals only $-0.14 \pm 0.02$ for the state reached by the real detector, the ideal detector allows to reach the value $-0.74 \pm 0.03$. Both these values belong to the nonclassical states as the corresponding values of the modified noise-reduction-parameter are smaller than $1\left(R_{n,+}=0.87 \pm 0.03, R_{n,+}^{\text {id }}=0.16 \pm 0.02\right)$. Also the real detector provides the marginal idler fields with the classical photon-number statistics close to the Poissonian one $\left(F_{n, \mathrm{i}_{1}}=1.03 \pm 0.09, F_{n, \mathrm{i}_{2}}=1.01 \pm 0.09\right)$. On the other hand, highly sub-Poissonian states arise for the ideal detector $\left(F_{n, \mathrm{i}_{1}}^{\mathrm{id}}=0.64 \pm 0.06, F_{n, \mathrm{i}_{2}}^{\mathrm{id}}=0.61 \pm 0.06\right)$. The $\mathrm{NCCa} C_{W}$ and $M_{W}$ assign the NCDs $\tau_{W}=0.06 \pm 0.02$ $\left(\tau_{C_{W}}=0.02 \pm 0.01, \tau_{M_{W}}=0.06 \pm 0.02\right)$ to the state obtained by the real detector and $0.40 \pm 0.01\left(\tau_{C_{W}}^{\mathrm{id}}=\right.$ $\left.0.38 \pm 0.01, \tau_{M_{W}}^{\mathrm{id}}=0.40 \pm 0.01\right)$ to the state provided by the ideal detector.

The decomposition of quasi-distribution $P_{\mathrm{ii}, s}\left(W_{i_{1}}, W_{i_{2}}\right)$ of the idler-fields integrated intensities related to an arbitrary $s$-ordering of field operators into the Laguerre polynomials allows to reconstruct the quasi-distribution $P_{\mathrm{ii}, s}$ from the corresponding photon-number distribution $p_{\mathrm{ii}}\left(n_{\mathrm{i}_{1}}, n_{\mathrm{i}_{2}}\right)$ [for details, see Appendix D]. The reconstructed quasi-distributions $P_{\mathrm{ii}}$ and $P_{\mathrm{ii}}^{\mathrm{id}}$ belonging to the analyzed fields are drawn in Figs. 4(c) and 4(d) for $s=0.1$ and $s=-0.15$, respectively. As there occur negative values in both graphs and according to the genuine definition of the nonclassicality [58, 59], the actual NCDs $\tau$ for the analyzed fields lie around 0.45 and 0.57 [see Eq. (10)], respectively. The areas with negative probability densities in the plane $\left(W_{i_{1}}, W_{i_{2}}\right)$ are typically located in the region between the point $\left(W_{i_{1}}, W_{i_{2}}\right)=(0,0)$ and the area where the maximal intensities of the quasi-distribution $P_{\mathrm{ii}, s}\left(W_{i_{1}}, W_{i_{2}}\right)$ occur [see the graph in Fig. 4(d)]. This resembles the behavior of $1 \mathrm{D}$ quasi-distributions of integrated intensities characterizing sub-Poissonian fields generated by photon-number-resolving postselection from TWBs [19].

The values of the NCDs $\tau$ indicated by negative values of the above quasi-distributions are considerably greater than those revealed by the $\mathrm{NCCa} C_{W}$ and $M_{W}$ based on the intensity moments, especially when the photonnumber distribution obtained by the real detector is analyzed. For this reason, we extend our analysis of the nonclassicality by considering the systems of NCCa involving the probabilities of photocount and photon-number distributions. Also in this case, the systems of NCCa $\bar{C}_{p}$ and $\bar{M}_{p}$ derived from the Cauchy-Schwarz inequality and the matrix approach, respectively, and described in detail in Appendix $\mathrm{C}$ proved the best performance. Moreover, to certain extent, they revealed the location of nonclassicality across the analyzed photon-number distributions, as demonstrated in Figs. $4(\mathrm{e}-\mathrm{h})$ showing the corresponding NCDs $\bar{\tau}$. The comparison of graphs in

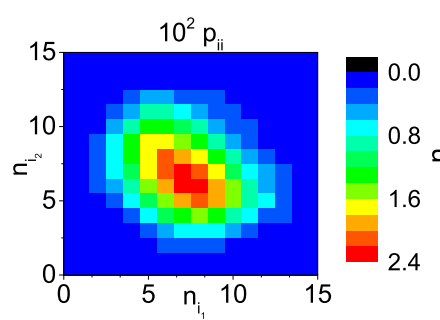

(a)

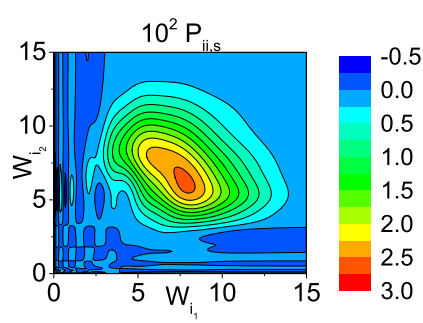

(c)

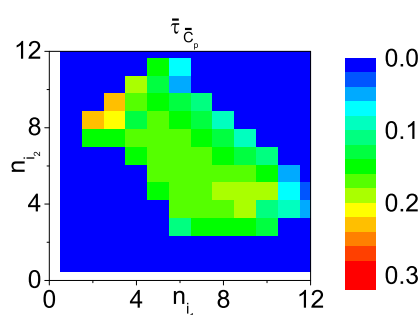

(e)

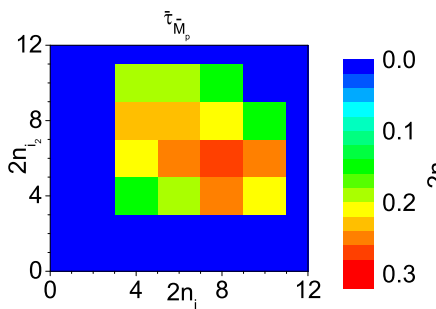

(g)

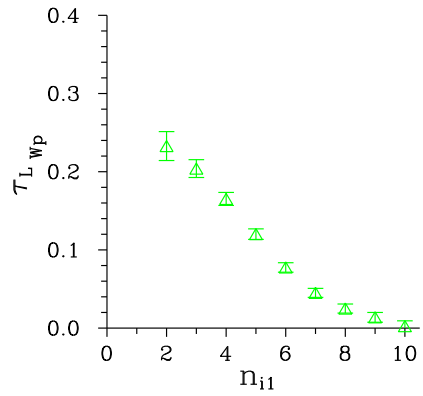

(i)

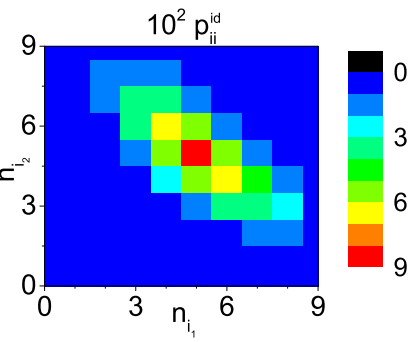

(b)

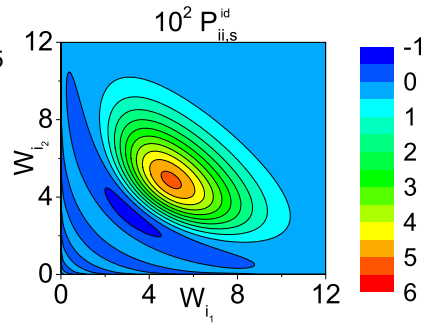

(d)

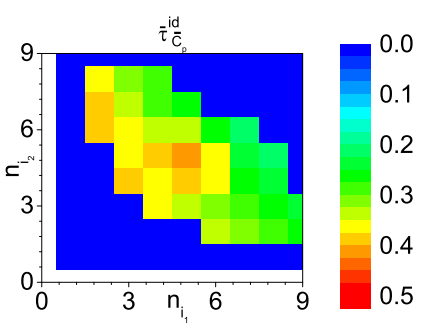

(f)

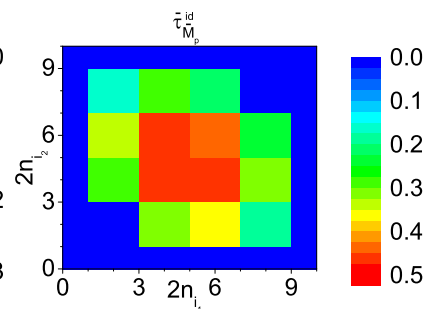

(h)

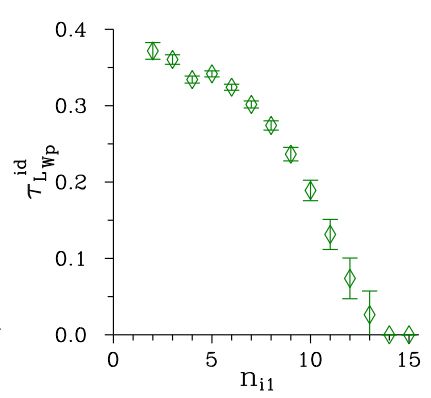

(j)

FIG. 4. (a,b) Photon-number distribution $p_{\mathrm{ii}}\left(n_{\mathrm{i}_{1}}, n_{\mathrm{i}_{2}}\right)$ with $(\mathrm{c}, \mathrm{d})$ the corresponding quasi-distribution $P_{\mathrm{ii}, s}\left(W_{i_{1}}, W_{i_{2}}\right)$ of integrated intensities and nonclassicality depths $\bar{\tau}$ of the $\operatorname{NCCa}(\mathrm{e}, \mathrm{f}) \bar{C}_{p}\left(n_{\mathrm{i}_{1}}, n_{\mathrm{i}_{2}}\right),(\mathrm{g}, \mathrm{h}) \bar{M}_{p}\left(n_{\mathrm{i}_{1}}, n_{\mathrm{i}_{2}}\right)$, and $(\mathrm{i}, \mathrm{j}) L_{W p}\left(n_{\mathrm{i}_{1}}\right)$ drawn as they depend on the numbers $n_{\mathrm{i}_{1}}$ and $n_{\mathrm{i}_{2}}$ of photons in the idler fields. The fields postselected by $c_{\mathrm{s}}=5$ signal photocounts (a,c,e,g,i) and $n_{\mathrm{s}}=10$ signal photons (b,d,f,h,j) are analyzed. In (c) $[(\mathrm{d})], s=0.1[s=-0.15]$ and the integrated intensities are expressed in the units of photon numbers. In $(e, g)[(f, h)]$, only the NCCa for which the mean value of the used probabilities is greater than 0.01 [0.02] are considered. In (i) $[(\mathrm{j})]$, isolated symbols (green $\triangle$ ) [dark green $\diamond]$ originate in $2 \mathrm{D}$ [3D] maximum-likelihood method. 
Figs. 4(e,f) with those in Figs. $4(\mathrm{~g}, \mathrm{~h})$ identifies the system of NCCa $\bar{M}_{p}$ as more powerful in quantifying the nonclassicality than the system of NCCa $\bar{C}_{p}$, similarly as in the case of their intensity-moment counterparts. The attained values of the NCDs $\bar{\tau}_{C_{p}}$ and $\bar{\tau}_{M_{p}}$ are greater than those reached by the NCCa $C_{W}$ and $M_{W}$ using the intensity moments. Considerable improvement occurs for both photon-number distributions $\left(\tau_{M_{W}}=0.06 \pm 0.02\right.$, $\left.\bar{\tau}_{M_{p}}^{\max }=0.27 ; \tau_{M_{W}}^{\mathrm{id}}=0.40 \pm 0.01, \bar{\tau}_{M_{p}}^{\mathrm{id}, \max }=0.46\right)$. The greatest values of the NCDs $\bar{\tau}_{M_{p}}$ are found in the central parts of the photon-number distributions [see Figs. $4(\mathrm{~g}, \mathrm{~h})]$.

In our opinion, the NCCa based on the intensity moments lose their power to resolve the nonclassicality compared to the NCCa with the probabilities in the process of averaging that smoothes out the local nonclassical features contained in the photon-number distributions. To support this explanation we analyze both photon-number distributions applying the hybrid criterion $L_{W p}$ in Eq. (9) that keeps the local 'resolution' in the first-idler-field photon number $n_{\mathrm{i}_{1}}$. The greatest achieved values of NCDs $\tau_{L_{W p}}$ and $\tau_{L_{W p}}^{\mathrm{id}}$ plotted in Figs. प(i) and $4(\mathrm{j})$, respectively, are smaller than the corresponding greatest values of the

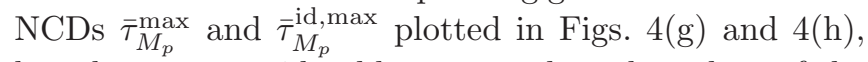
but they are considerably greater than the values of the corresponding NCDs $\tau_{M_{W}}$ and $\tau_{M_{W}}^{\mathrm{id}}$.

\section{CONCLUSIONS}

Using postselection by a photon-number-resolving detector and two twin beams of similar intensities, we have experimentally generated the fields with increasing intensities that are endowed with anticorrelations in photonnumber fluctuations. They even exhibit the marginal sub-Poissonian photon-number statistics under suitable conditions. Properties of the experimentally generated postselected states were monitored by two additional photon-number-resolving detectors. The obtained experimental data were reconstructed in parallel by the maximum-likelihood approach and by considering a suitable Gaussian fit. The nonclassicality of the observed postselected fields was evidenced by the determination of the corresponding quasi-distributions of integrated intensities with negative values as well as by several types of the nonclassicality criteria and the accompanying nonclassicality depths. Whereas the quasi-distributions of integrated intensities are natural identifiers of the nonclassicality, the ability of the nonclassicality criteria to resolve the nonclassicality decreases with their decreasing 'resolution' (in turn, criteria based on the probabilities, hybrid criteria and criteria using the intensity moments). Specific properties of the generated states are appealing in quantum metrology: The measurement of two-photon absorption cross-sections beyond the shot-noise-limit because of the sub-Poissonian character of both fields and anticorrelations in photon-number fluctuations serves as an example. The properties of the investigated states are also attractive for two-photon excitations of molecules and other material systems.

\section{ACKNOWLEDGMENTS}

The authors thank GA ČR projects No. 18-08874S (V.M., R.M., O.H.) and No. 18-22102S (J.P.). They also acknowledge the support from MŠMT ČR (project No. CZ.02.1.01/0.0/0.0/16_019/0000754).

\section{Appendix A: Multi-mode Gaussian fields and their reconstruction}

The mechanism of generation of the analyzed optical field suggests the following analytical structure for its description. The optical field may be considered as composed of two ideal multi-mode TWBs and three independent multi-mode thermal (Gaussian) noisy fields. Spontaneous character of parametric down-conversion suggests the photon-number distribution $p_{\mathrm{p}_{j}}\left(n_{\mathrm{s}_{j}}, n_{\mathrm{i}_{j}}\right)$ for TWB $j, j=1,2$, in the multi-mode Gaussian form with $M_{\mathrm{p}_{j}}$ modes and $B_{\mathrm{p}_{j}}$ mean photon-pairs per mode

$$
p_{\mathrm{p}_{j}}\left(n_{\mathrm{s}_{j}}, n_{\mathrm{i}_{j}}\right)=\delta_{n_{\mathrm{s}_{j}}, n_{\mathrm{i}_{j}}} p^{\mathrm{M}-\mathrm{R}}\left(n_{\mathrm{s}_{j}} ; M_{\mathrm{p}_{j}}, B_{\mathrm{p}_{j}}\right) .
$$

The multi-mode thermal Mandel-Rice distribution $p^{\mathrm{M}-\mathrm{R}}$ for an $M$-mode field with each mode having on average $B$ photons is given as:

$$
p^{\mathrm{M}-\mathrm{R}}(n ; M, B)=\frac{\Gamma(n+M)}{n ! \Gamma(M)} \frac{B^{n}}{(1+B)^{n+M}} .
$$

In Eqs. A1 and (A2), the Kronecker symbol $\delta_{n_{\mathrm{s}}, n_{i}}$ and the gamma function $\Gamma$ are used.

A 3D photon-number distribution $p_{\mathrm{p}}$ of the ideally paired part of the studied optical field is expresses as:

$$
p_{\mathrm{p}}\left(n_{\mathrm{s}}, n_{\mathrm{i}_{1}}, n_{\mathrm{i}_{2}}\right)=\sum_{n_{\mathrm{s}_{1}}=0}^{n_{\mathrm{s}}} p_{\mathrm{p}_{1}}\left(n_{\mathrm{s}_{1}}, n_{\mathrm{i}_{1}}\right) p_{\mathrm{p}_{2}}\left(n_{\mathrm{s}}-n_{\mathrm{s}_{1}}, n_{\mathrm{i}_{2}}\right) .
$$

We assume the photon-number distribution $p_{\mathrm{n}_{\mathrm{s}}}$ of the noise in the combined signal field in the form of Eq. A2 with $M_{\mathrm{n}_{\mathrm{s}}}$ modes each having on average $B_{\mathrm{n}_{\mathrm{s}}}$ noisy photons. Similar assumption is made for the photon-number distribution $p_{n_{\mathrm{i}_{\mathrm{j}}}}$ of the $j$-th idler field whose noise is distributed into $M_{\mathrm{n}_{\mathrm{i}_{\mathrm{j}}}}$ modes each populated with $B_{\mathrm{n}_{\mathrm{i}_{\mathrm{j}}}}$ mean photons, $j=1,2$. Three-fold convolution of the ideally paired photon-number distribution $p_{\mathrm{p}}$ with three noisy photon-number distributions then leaves us with the photon-number distribution $p$ appropriate for the analyzed optical field:

$$
p\left(n_{\mathrm{s}}, n_{\mathrm{i}_{1}}, n_{\mathrm{i}_{2}}\right)=\sum_{l_{\mathrm{s}}=0}^{n_{\mathrm{s}}} p_{\mathrm{n}_{\mathrm{s}}}\left(n_{\mathrm{s}}-l_{\mathrm{s}}\right) \sum_{l_{\mathrm{i}_{1}}=0}^{n_{\mathrm{i}_{1}}} p_{\mathrm{n}_{\mathrm{i}_{1}}}\left(n_{\mathrm{i}_{1}}-l_{\mathrm{i}_{1}}\right)
$$




$$
\times \sum_{l_{\mathrm{i}_{2}}=0}^{n_{\mathrm{i}_{2}}} p_{\mathrm{n}_{\mathrm{i}_{2}}}\left(n_{\mathrm{i}_{2}}-l_{\mathrm{i}_{2}}\right) p_{\mathrm{p}}\left(l_{\mathrm{s}}, l_{\mathrm{i}_{1}}, l_{\mathrm{i}_{2}}\right) .
$$

The photon-number moments $\left\langle n_{\mathrm{s}}^{k_{\mathrm{s}}} n_{\mathrm{i}_{1}}^{k_{\mathrm{i}_{1}}} n_{\mathrm{i}_{2}}^{k_{\mathrm{i}_{2}}}\right\rangle$ corresponding to the photon-number distribution $p$ in Eq. (A4) are determined as follows:

$$
\left\langle n_{\mathrm{s}}^{k_{\mathrm{s}}} n_{\mathrm{i}_{1}}^{k_{\mathrm{i}_{1}}} n_{\mathrm{i}_{2}}^{k_{\mathrm{i}_{2}}}\right\rangle=\sum_{n_{\mathrm{s}}, n_{\mathrm{i}_{1}}, n_{\mathrm{i}_{2}}=0}^{\infty} n_{\mathrm{s}}^{k_{\mathrm{s}}} n_{\mathrm{i}_{1}}^{k_{\mathrm{i}_{1}}} n_{\mathrm{i}_{2}}^{k_{\mathrm{i}_{2}}} p\left(n_{\mathrm{s}}, n_{\mathrm{i}_{1}}, n_{\mathrm{i}_{2}}\right) .
$$

The (integrated-) intensity moments $\left\langle W_{\mathrm{s}}^{k_{\mathrm{s}}} W_{\mathrm{i}_{1}}^{k_{\mathrm{i}_{1}}} W_{\mathrm{i}_{2}}^{k_{\mathrm{i}_{2}}}\right\rangle$, that are the normally-ordered photon-number moments, are derived from the above photon-number moments using the Stirling numbers $S$ of the first kind [60]:

$$
\begin{aligned}
\left\langle W_{\mathrm{s}}^{k_{\mathrm{s}}} W_{\mathrm{i}_{1}}^{k_{\mathrm{i}_{1}}} W_{\mathrm{i}_{2}}^{k_{\mathrm{i}_{2}}}\right\rangle & =\sum_{l_{\mathrm{s}}=0}^{k_{\mathrm{s}}} S\left(k_{\mathrm{s}}, l_{\mathrm{s}}\right) \sum_{l_{\mathrm{i}_{1}}=0}^{k_{\mathrm{i}_{1}}} S\left(k_{\mathrm{i}_{1}}, l_{\mathrm{i}_{1}}\right) \\
& \times \sum_{l_{\mathrm{i}_{2}}=0}^{k_{\mathrm{i}_{2}}} S\left(k_{\mathrm{i}_{2}}, l_{\mathrm{i}_{2}}\right)\left\langle n_{\mathrm{s}}^{l_{\mathrm{s}}} n_{\mathrm{i}_{1}}^{l_{\mathrm{i}_{1}}} n_{\mathrm{i}_{2}}^{l_{\mathrm{i}_{2}}}\right\rangle .
\end{aligned}
$$

The inverse relation to that in Eq. A6 relies on the Stirling numbers of the second kind. We note that we have the following relations between the intensity moments and number $M$ of modes together with their mean photon numbers $B$ for a multi-mode thermal field:

$$
B=\frac{\left\langle(\Delta W)^{2}\right\rangle}{\langle W\rangle}, \quad M=\frac{\langle W\rangle^{2}}{\left\langle(\Delta W)^{2}\right\rangle} ;
$$

$\Delta W \equiv W-\langle W\rangle$.

In the experiment, we detect the photocount numbers $c$, i.e. the numbers of photoelectons excited by the absorbed photons. Multiple realizations of the measurement then give us the experimental photocount histogram $f$ determined in Eq. (3) and the accompanying photocount moments $\left\langle c_{\mathrm{s}}^{k_{\mathrm{s}}} c_{\mathrm{i}_{1}}^{k_{\mathrm{i}_{1}}} c_{\mathrm{i}_{2}}^{k_{\mathrm{i}_{2}}}\right\rangle$,

$$
\left\langle c_{\mathrm{s}}^{k_{\mathrm{s}}} c_{\mathrm{i}_{1}}^{k_{\mathrm{i}_{1}}} c_{\mathrm{i}_{2}}^{k_{\mathrm{i}_{2}}}\right\rangle=\sum_{c_{\mathrm{s}}, c_{\mathrm{i}_{1}}, c_{\mathrm{i}_{2}}=0}^{\infty} c_{\mathrm{s}}^{k_{\mathrm{s}}} c_{\mathrm{i}_{1}}^{k_{\mathrm{i}_{1}}} c_{\mathrm{i}_{2}}^{k_{\mathrm{i}_{2}}} f\left(c_{\mathrm{s}}, c_{\mathrm{i}_{1}}, c_{\mathrm{i}_{2}}\right) .
$$

Similarly as the intensity moments $\left\langle W_{\mathrm{s}}^{k_{\mathrm{s}}} W_{\mathrm{i}_{1}}^{k_{\mathrm{i}_{1}}} W_{\mathrm{i}_{2}}^{k_{\mathrm{i}_{2}}}\right\rangle$ are assigned to the photon-number moments $\left\langle n_{\mathrm{s}}^{l_{s}} n_{\mathrm{i}_{1}}^{l_{1_{1}}} n_{\mathrm{i}_{2}}^{l_{\mathrm{i}_{2}}}\right\rangle$, we may assign the intensity moments $\left\langle\mathcal{W}_{\mathrm{s}}^{k_{\mathrm{s}}} \mathcal{W}_{\mathrm{i}_{1}}^{k_{\mathrm{i}_{1}}} \mathcal{W}_{\mathrm{i}_{2}}^{k_{\mathrm{i}_{2}}}\right\rangle_{E}$ to the photocount moments $\left\langle c_{\mathrm{s}}^{l_{\mathrm{s}}} c_{\mathrm{i}_{1}}^{l_{\mathrm{i}_{1}}} c_{\mathrm{i}_{2}}^{l_{\mathrm{i}_{2}}}\right\rangle$ using the relations in Eq. (A6). The photocount moments $\left\langle c_{\mathrm{s}}^{l_{\mathrm{s}}} c_{\mathrm{i}_{1}}^{l_{\mathrm{i}_{1}}} c_{\mathrm{i}_{2}}^{l_{\mathrm{i}_{2}}}\right\rangle$ as well as the intensity moments $\left\langle\mathcal{W}_{\mathrm{s}}^{k_{s}} \mathcal{W}_{\mathrm{i}_{1}}^{k_{\mathrm{i}_{1}}} \mathcal{W}_{\mathrm{i}_{2}}^{k_{\mathrm{i}_{2}}}\right\rangle_{E}$ are directly available from the experimental data and so they form a natural basis for the reconstruction of the above Gaussian form of the studied field.

Description of the response of a PNRD is also needed when making the reconstruction. An iCCD camera, used in our experiment, is characterized by detection efficiency $\eta$, dark-count rate $D \equiv d / N$ per pixel and number $N$ of active pixels that determine the corresponding detection matrix $T(c, n)$ introduced in Eq. (2) in the following form 61]:

$$
\begin{gathered}
T(c, n)=\left(\begin{array}{c}
N \\
c
\end{array}\right)(1-D)^{N}(1-\eta)^{n}(-1)^{c} \\
\times \sum_{l=0}^{c}\left(\begin{array}{l}
c \\
l
\end{array}\right) \frac{(-1)^{l}}{(1-D)^{l}}\left(1+\frac{l}{N} \frac{\eta}{1-\eta}\right)^{n} .
\end{gathered}
$$

For the reconstruction, we have at our disposal the experimental 3D photocount histogram $f$. From this histogram, we conveniently determine the following nine experimental intensity moments with sufficiently high precision: $\left\langle\mathcal{W}_{\mathrm{s}}\right\rangle_{E},\left\langle\mathcal{W}_{\mathrm{i}_{j}}\right\rangle_{E},\left\langle\left(\Delta \mathcal{W}_{\mathrm{s}}\right)^{2}\right\rangle_{E},\left\langle\left(\Delta \mathcal{W}_{\mathrm{i}_{j}}\right)^{2}\right\rangle_{E}$, $\left\langle\Delta \mathcal{W}_{\mathrm{s}} \Delta \mathcal{W}_{\mathrm{i}_{j}}\right\rangle_{E}$, and $\left\langle\Delta \mathcal{W}_{\mathrm{i}_{1}} \Delta \mathcal{W}_{\mathrm{i}_{2}}\right\rangle_{E}, j=1,2$. On the other hand, the multi-mode Gaussian optical field is characterized by ten parameters, five parameters give the numbers of modes $\left(M_{\mathrm{p}_{j}}, M_{\mathrm{n}_{\mathrm{s}}}, M_{\mathrm{n}_{\mathrm{i}}}, j=1,2\right)$ in different components of the field and five parameters characterize the mean photon (-pair) numbers in each mode $\left(B_{\mathrm{p}_{j}}\right.$, $\left.B_{\mathrm{n}_{\mathrm{s}}}, B_{\mathrm{n}_{\mathrm{i}}}, j=1,2\right)$. Moreover, we need to know the detection efficiencies for each detected field $\left(\eta_{\mathrm{s}}, \eta_{\mathrm{i}_{1}}, \eta_{\mathrm{i}_{2}}\right)$.

Detailed analysis of the used experimental setup reveals that the detection efficiencies $\eta_{\mathrm{i}_{1}}$ and $\eta_{\mathrm{i}_{2}}$ cannot be determined independently with sufficient precision. This is related to the fact that no photon pairs occur directly in the first and the second idler fields. For this reason, we assume in our analysis that they equal $\left(\eta_{\mathrm{i}_{1}}=\eta_{\mathrm{i}_{2}} \equiv \eta_{\mathrm{i}}\right)$. Under this assumption we can accomplish the reconstruction in two subsequent steps.

First, we combine together the intensity moments of both idler fields to arrive at the moments characterizing the common idler field:

$$
\begin{aligned}
\left\langle\mathcal{W}_{\mathrm{i}}\right\rangle_{E}= & \left\langle\mathcal{W}_{\mathrm{i}_{1}}\right\rangle_{E}+\left\langle\mathcal{W}_{\mathrm{i}_{2}}\right\rangle_{E} \\
\left\langle\left(\Delta \mathcal{W}_{\mathrm{i}}\right)^{2}\right\rangle_{E}= & \left\langle\left(\Delta \mathcal{W}_{\mathrm{i}_{1}}\right)^{2}\right\rangle_{E}+2\left\langle\Delta \mathcal{W}_{\mathrm{i}_{1}} \Delta \mathcal{W}_{\mathrm{i}_{2}}\right\rangle_{E} \\
& +\left\langle\left(\Delta \mathcal{W}_{\mathrm{i}_{2}}\right)^{2}\right\rangle_{E} \\
\left\langle\Delta \mathcal{W}_{\mathrm{s}} \Delta \mathcal{W}_{\mathrm{i}}\right\rangle_{E}= & \left\langle\Delta \mathcal{W}_{\mathrm{s}} \Delta \mathcal{W}_{\mathrm{i}_{1}}\right\rangle_{E}+\left\langle\Delta \mathcal{W}_{\mathrm{s}} \Delta \mathcal{W}_{\mathrm{i}_{2}}\right\rangle_{E} .
\end{aligned}
$$

Then we apply the reconstruction method for a multimode Gaussian TWB composed of the combined signal and combined idler fields that has been developed in 62]. This provides us the intensity moments $\left\langle W_{\mathrm{p}}\right\rangle$ and $\left\langle\left(\Delta W_{\mathrm{p}}\right)^{2}\right\rangle$ of the combined ideally paired field and intensity moments $\left\langle W_{\mathrm{n}_{\mathrm{s}}}\right\rangle,\left\langle W_{\mathrm{n}_{\mathrm{i}}}\right\rangle,\left\langle\left(\Delta W_{\mathrm{n}_{\mathrm{s}}}\right)^{2}\right\rangle$, and $\left\langle\left(\Delta W_{\mathrm{n}_{\mathrm{i}}}\right)^{2}\right\rangle$ of the noise signal and idler fields as well as the detection efficiencies $\eta_{\mathrm{s}}$ and $\eta_{\mathrm{i}}$.

In the second step, we determine the remaining intensity moments $\left\langle W_{\mathrm{p}_{j}}\right\rangle$ and $\left\langle\left(\Delta W_{\mathrm{p}_{j}}\right)^{2}\right\rangle$ belonging to the paired components as well as the intensity moments $\left\langle W_{\mathrm{i}_{j}}\right\rangle$ and $\left\langle\left(\Delta W_{\mathrm{i}_{j}}\right)^{2}\right\rangle$ of the noise idler fields, $j=1,2$. For this purpose, we write the following ten linear relations among the looked-for intensity moments:

$$
\left\langle W_{\mathrm{p}_{j}}\right\rangle+\left\langle W_{\mathrm{n}_{\mathrm{i}_{j}}}\right\rangle=\left\langle\mathcal{W}_{\mathrm{i}_{j}}\right\rangle_{E} / \eta_{\mathrm{i}},
$$




$$
\begin{aligned}
\left\langle\left(\Delta W_{\mathrm{p}_{j}}\right)^{2}\right\rangle+\left\langle\left(\Delta W_{\mathrm{i}_{j}}\right)^{2}\right\rangle & =\left\langle\left(\Delta \mathcal{W}_{\mathrm{i}_{j}}\right)^{2}\right\rangle_{E} / \eta_{\mathrm{i}}^{2}, \\
\left\langle W_{\mathrm{p}_{j}}\right\rangle+\left\langle\left(\Delta W_{\mathrm{p}_{j}}\right)^{2}\right\rangle & =\left\langle\Delta \mathcal{W}_{\mathrm{i}_{j}} \Delta \mathcal{W}_{\mathrm{s}}\right\rangle_{E} /\left(\eta_{\mathrm{i}} \eta_{\mathrm{s}}\right), \\
\left\langle W_{\mathrm{p}_{1}}\right\rangle+\left\langle W_{\mathrm{p}_{2}}\right\rangle & =\left\langle W_{\mathrm{p}}\right\rangle, \\
\left\langle W_{\mathrm{n}_{\mathrm{i}_{1}}}\right\rangle+\left\langle W_{\mathrm{n}_{\mathrm{i}_{2}}}\right\rangle & =\left\langle W_{\mathrm{n}_{\mathrm{i}}}\right\rangle, \\
\left\langle\left(\Delta W_{\mathrm{p}_{1}}\right)^{2}\right\rangle+\left\langle\left(\Delta W_{\mathrm{p}_{2}}\right)^{2}\right\rangle & =\left\langle\left(\Delta W_{\mathrm{p}}\right)^{2}\right\rangle, \\
\left\langle\left(\Delta W_{\mathrm{n}_{\mathrm{i}_{1}}}\right)^{2}\right\rangle+\left\langle\left(\Delta W_{\mathrm{n}_{\mathrm{i}_{2}}}\right)^{2}\right\rangle & =\left\langle\left(\Delta W_{\mathrm{n}_{\mathrm{i}}}\right)^{2}\right\rangle .
\end{aligned}
$$

Whereas the first six relations in Eq. (A11) contain the original experimental intensity moments, the remaining four relations are based upon the intensity moments obtained in the first step.

Detailed analysis of the linear relations in Eq. A11) reveals that only seven out of them are independent. As we have eight independent intensity moments to be determined, we choose one intensity moment as a free parameter and derive the remaining seven ones using the relations in Eq. (A11). We may conveniently choose, e.g., the moment $\left\langle\left(\Delta W_{\mathrm{p}_{1}}\right)^{2}\right\rangle$ and express the remaining moments as linear combinations of this moment, the experimental intensity moments and the moments known from the first step. We may proceed, e.g., along the following lines: $\left\langle\left(\Delta W_{\mathrm{p}_{1}}\right)^{2}\right\rangle \rightarrow\left\langle W_{\mathrm{p}_{1}}\right\rangle \rightarrow\left\langle W_{\mathrm{p}_{2}}\right\rangle \rightarrow\left\langle\left(\Delta W_{\mathrm{p}_{2}}\right)^{2}\right\rangle$, $\left\langle W_{\mathrm{p}_{j}}\right\rangle \rightarrow\left\langle W_{\mathrm{n}_{\mathrm{i}_{j}}}\right\rangle,\left\langle\left(\Delta W_{\mathrm{p}_{j}}\right)^{2}\right\rangle \rightarrow\left\langle\left(\Delta W_{\mathrm{n}_{\mathrm{i}_{j}}}\right)^{2}\right\rangle, j=1,2$. We note that the allowed values of the intensity moment $\left\langle\left(\Delta W_{\mathrm{p}_{1}}\right)^{2}\right\rangle$ fulfill:

$$
\begin{aligned}
&\left\langle\left(\Delta W_{\mathrm{p}_{1}}\right)^{2}\right\rangle \in(0, \min \left\{\left\langle\left(\Delta \mathcal{W}_{\mathrm{i}_{1}}\right)^{2}\right\rangle_{E} / \eta_{\mathrm{i}}^{2},\right. \\
&\left.\left.\left\langle\Delta \mathcal{W}_{\mathrm{i}_{1}} \Delta \mathcal{W}_{\mathrm{s}}\right\rangle_{E} /\left(\eta_{\mathrm{i}} \eta_{\mathrm{s}}\right)\right\}\right) .
\end{aligned}
$$

For given set of the values of the intensity moments $\left\langle W_{\mathrm{p}_{j}}\right\rangle,\left\langle\left(\Delta W_{\mathrm{p}_{j}}\right)^{2}\right\rangle,\left\langle W_{\mathrm{n}_{\mathrm{i}_{j}}}\right\rangle,\left\langle\left(\Delta W_{\mathrm{n}_{\mathrm{i}_{j}}}\right)^{2}\right\rangle, j=1,2,\left\langle W_{n_{\mathrm{s}}}\right\rangle$, and $\left\langle\left(\Delta W_{n_{\mathrm{s}}}\right)^{2}\right\rangle$ we derive the numbers $M_{\mathrm{p}_{j}}, M_{\mathrm{n}_{\mathrm{i}}}, j=$ 1,2 , and $M_{n_{\mathrm{s}}}$ of modes and mean photon (-pair) numbers $B_{\mathrm{p}_{j}}, B_{\mathrm{n}_{\mathrm{i}_{j}}}, j=1,2$, and $B_{n_{\mathrm{s}}}$ using Eqs. A77. Then, we reconstruct the $3 \mathrm{D}$ photon number distribution $p\left(n_{\mathrm{s}}, n_{\mathrm{i}_{1}}, n_{\mathrm{i}_{2}}\right)$ in Eq. A3 and arrive at the theoretical 3D photocount histogram $f^{\text {th }}\left(c_{\mathrm{s}}, c_{\mathrm{i}_{1}}, c_{\mathrm{i}_{2}}\right)$ by applying Eq. (3) together with the detection matrix in Eq. (A9). The optimal values of numbers of modes and mean photon (-pair) numbers are set such that they minimize the declination function $\mathcal{D}$ between the theoretical and experimental histograms:

$$
\mathcal{D}=\sqrt{\sum_{c_{\mathrm{s}}, c_{\mathrm{i}_{1}}, c_{\mathrm{i}_{2}}=0}^{\infty}\left[f^{\mathrm{th}}\left(c_{\mathrm{s}}, c_{\mathrm{i}_{1}}, c_{\mathrm{i}_{2}}\right)-f\left(c_{\mathrm{s}}, c_{\mathrm{i}_{1}}, c_{\mathrm{i}_{2}}\right)\right]^{2}} .
$$

\section{Appendix B: Maximum-likelihood reconstruction of $2 \mathrm{D}$ and $3 \mathrm{D}$ photon-number distributions}

The 3D photon-number distribution $p\left(n_{\mathrm{s}}, n_{\mathrm{i}_{1}}, n_{\mathrm{i}_{2}}\right)$ of the original optical field used in the experiment is obtained from the experimental photocount histogram $f\left(c_{\mathrm{s}}, c_{\mathrm{i}_{1}}, c_{\mathrm{i}_{2}}\right)$ by inverting the linear relations expressed in Eq. (31). The maximum-likelihood method [63, 64] provides us the following iteration procedure that reveals the photon-number distribution $p\left(n_{\mathrm{s}}, n_{\mathrm{i}_{1}}, n_{\mathrm{i}_{2}}\right)$ as a steady state of the following iteration procedure:

$$
\begin{aligned}
& p^{(j+1)}\left(n_{\mathrm{s}}, n_{\mathrm{i}_{1}}, n_{\mathrm{i}_{2}}\right)=\sum_{c_{\mathrm{s}}, c_{\mathrm{i}_{1}}, c_{\mathrm{i}_{2}}=0}^{\infty} F^{(j)}\left(c_{\mathrm{s}}, c_{\mathrm{i}_{1}}, c_{\mathrm{i}_{2}}\right) T_{\mathrm{s}}\left(c_{\mathrm{s}}, n_{\mathrm{s}}\right) \\
& \times T_{\mathrm{i}_{1}}\left(c_{\mathrm{i}_{1}}, n_{\mathrm{i}_{1}}\right) T_{\mathrm{i}_{2}}\left(c_{\mathrm{i}_{2}}, n_{\mathrm{i}_{2}}\right), \\
& F^{(j)}\left(c_{\mathrm{s}}, c_{\mathrm{i}_{1}}, c_{\mathrm{i}_{2}}\right)=f\left(c_{\mathrm{s}}, c_{\mathrm{i}_{1}}, c_{\mathrm{i}_{2}}\right)\left[\sum_{n_{\mathrm{s}}^{\prime}, n_{\mathrm{i}_{1}}^{\prime}, n_{\mathrm{i}_{2}}^{\prime}=0}^{\infty} T_{\mathrm{s}}\left(c_{\mathrm{s}}, n_{\mathrm{s}}^{\prime}\right)\right. \\
& \left.\times T_{\mathrm{i}_{1}}\left(c_{\mathrm{i}_{1}}, n_{\mathrm{i}_{1}}^{\prime}\right) T_{\mathrm{i}_{2}}\left(c_{\mathrm{i}_{2}}, n_{\mathrm{i}_{2}}^{\prime}\right) p^{(j)}\left(n_{\mathrm{s}}^{\prime}, n_{\mathrm{i}_{1}}^{\prime}, n_{\mathrm{i}_{2}}^{\prime}\right)\right]^{-1}, \\
& j=0,1, \ldots \text {. }
\end{aligned}
$$

Similarly, the 2D photon-number distributions $p_{\mathrm{ii}}\left(n_{\mathrm{i}_{1}}, n_{\mathrm{i}_{2}} ; c_{\mathrm{s}}\right)$ given in Eq. (2) and belonging to the field postselected by detecting $c_{\mathrm{s}}$ signal photocounts can be reconstructed by the maximum-likelihood method from the conditional experimental photocount histograms $f_{\mathrm{ii}}\left(c_{\mathrm{i}_{1}}, c_{\mathrm{i}_{2}} ; c_{\mathrm{s}}\right) \equiv f\left(c_{\mathrm{s}}, c_{\mathrm{i}_{1}}, c_{\mathrm{i}_{2}}\right) / \sum_{c_{\mathrm{i}_{1}}^{\prime}, c_{\mathrm{i}_{2}}^{\prime}=0}^{\infty} f\left(c_{\mathrm{s}}, c_{\mathrm{i}_{1}}^{\prime}, c_{\mathrm{i}_{2}}^{\prime}\right)$. We arrive at the following iteration procedure in this case:

$$
\begin{aligned}
p_{\mathrm{ii}}^{(j+1)}\left(n_{\mathrm{i}_{1}}, n_{\mathrm{i}_{2}} ; c_{\mathrm{s}}\right) & =\sum_{c_{\mathrm{i}_{1}}, c_{\mathrm{i}_{2}}=0}^{\infty} F_{\mathrm{ii}}^{(j)}\left(c_{\mathrm{i}_{1}}, c_{\mathrm{i}_{2}} ; c_{\mathrm{s}}\right) T_{\mathrm{i}_{1}}\left(c_{\mathrm{i}_{1}}, n_{\mathrm{i}_{1}}\right) \\
& \times T_{\mathrm{i}_{2}}\left(c_{\mathrm{i}_{2}}, n_{\mathrm{i}_{2}}\right), \\
F_{\mathrm{ii}}^{(j)}\left(c_{\mathrm{i}_{1}}, c_{\mathrm{i}_{2}} ; c_{\mathrm{s}}\right) & =f_{i i}\left(c_{\mathrm{i}_{1}}, c_{\mathrm{i}_{2}} ; c_{\mathrm{s}}\right)\left[\sum_{n_{\mathrm{i}_{1}}^{\prime}, n_{\mathrm{i}_{2}}^{\prime}=0}^{\infty} T_{\mathrm{i}_{1}}\left(c_{\mathrm{i}_{1}}, n_{\mathrm{i}_{1}}^{\prime}\right)\right. \\
\times & \left.T_{\mathrm{i}_{2}}\left(c_{\mathrm{i}_{2}}, n_{\mathrm{i}_{2}}^{\prime}\right) p_{\mathrm{ii}}^{(j)}\left(n_{\mathrm{i}_{1}}^{\prime}, n_{\mathrm{i}_{2}}^{\prime} ; c_{\mathrm{s}}\right)\right]^{-1}, j=0,1, \ldots
\end{aligned}
$$

\section{Appendix C: Identification of the nonclassicality}

For the analyzed postselected 2D idler fields, the NCCa $C_{K}^{L}$ derived from the Cauchy-Schwarz inequality and the NCCa $M_{J K L}$ originating in non-negative quadratic forms 38] of three variables conveniently written in the matrix form [57, 65, 66] have been found the most powerful:

$$
\begin{gathered}
C_{K}^{L}=\left\langle W^{L}\right\rangle\left\langle W^{2 K-L}\right\rangle-\left\langle W^{K}\right\rangle^{2}<0, \\
K \geq 0,2 K \geq L \geq 0 \\
M_{J K L}=\operatorname{det}\left\langle\left[\begin{array}{ccc}
W^{2 J} & W^{J+K} & W^{J+L} \\
W^{K+J} & W^{2 K} & W^{K+L} \\
W^{L+J} & W^{L+K} & W^{2 L}
\end{array}\right]\right\rangle<0, \\
J, K, L \geq 0 .
\end{gathered}
$$

In Eqs. (C1) and (C2), we use the notation with vector indices $K \equiv\left(k_{\mathrm{i}_{1}}, k_{\mathrm{i}_{2}}\right)$ in which $W^{K} \equiv W_{\mathrm{i}_{1}}^{k_{\mathrm{i}_{1}}} W_{\mathrm{i}_{2}}^{k_{\mathrm{i}_{2}}}$ and $K ! \equiv k_{\mathrm{i}_{1}} ! k_{\mathrm{i}_{2}} !$

The NCCa $C_{K}^{L}$ and $M_{J K L}$ based on the intensity moments are translated into the corresponding NCCa 
$\bar{C}_{K}^{L}$ and $\bar{M}_{J K L}$ written for the probabilities of photonnumber (photocount) distributions $p\left(k_{\mathrm{i}_{1}}, k_{\mathrm{i}_{2}}\right) \equiv p(K)$ [56, 67 70] using the mapping originating in the Mandel detection formula [2, 55]:

$$
\left\langle W^{K}\right\rangle \longleftarrow K ! p(K) / p(0,0)
$$

We note that the mapping (C3) assigns photon numbers and the accompanying probabilities to the powers of intensity moments. The NCCa for probabilities indicate not only the global nonclassicality of an analyzed field, they may also provide the information about the location of the nonclassicality across the profile of photon-number (photocount) distribution [57]. This can be accomplished by applying the following NCCa $\bar{C}_{p}(K)$ and $\bar{M}_{p}(K)$ that involve the above NCCa $\bar{C}_{K}^{L}$ and $\bar{M}_{J K L}$ with the indices obeying specific conditions:

$$
\begin{aligned}
\bar{C}_{p}(K) & =\min _{L,|K-L| \leq 1}\left\{\bar{C}_{K}^{L}\right\}, \\
\bar{M}_{p}(K) & =\min _{J, L,|J-K| \leq 1,|L-K| \leq 1}\left\{\bar{M}_{J K L}\right\},
\end{aligned}
$$

and $|K-L| \leq 1$ means that both conditions $\left|k_{\mathrm{i}_{j}}-l_{\mathrm{i}_{j}}\right| \leq 1$ for $j=1,2$ are fulfilled.

\section{Appendix D: Reconstruction of quasi-distributions of integrated intensities}

An $s$-ordered quasi-distribution $P_{\mathrm{ii}, s}\left(W_{\mathrm{i}_{1}}, W_{\mathrm{i}_{2}}\right)$ of the idler-fields integrated intensities $W_{\mathrm{i}_{1}}$ and $W_{\mathrm{i}_{2}}$ corresponding to a $2 \mathrm{D}$ idler-fields photon-number distribution $p_{\mathrm{ii}}\left(n_{\mathrm{i}_{1}}, n_{\mathrm{i}_{2}}\right)$ is obtained using the following formula [55]:

$$
\begin{aligned}
P_{\mathrm{ii}, s}\left(W_{\mathrm{i}_{1}},\right. & \left.W_{\mathrm{i}_{2}}\right)=\frac{4}{(1-s)^{2}} \exp \left(-\frac{2\left(W_{\mathrm{i}_{1}}+W_{\mathrm{i}_{2}}\right)}{1-s}\right) \\
& \times \sum_{n_{\mathrm{i}_{1}}, n_{\mathrm{i}_{2}}=0}^{\infty} \frac{p_{\mathrm{ii}_{1}}\left(n_{\mathrm{i}_{1}}, n_{\mathrm{i}_{2}}\right)}{n_{\mathrm{i}_{1}} ! n_{\mathrm{i}_{2}} !}\left(\frac{s+1}{s-1}\right)^{n_{\mathrm{i}_{1}}+n_{\mathrm{i}_{2}}} \\
& \times L_{n_{\mathrm{i}_{1}}}\left(\frac{4 W_{\mathrm{i}_{1}}}{1-s^{2}}\right) L_{n_{\mathrm{i}_{2}}}\left(\frac{4 W_{\mathrm{i}_{2}}}{1-s^{2}}\right) .
\end{aligned}
$$

In Eq. (D1), the symbol $L_{k}$ stands for the Laguerre polynomials [1].
[1] R. W. Boyd, Nonlinear Optics, 2nd edition (Academic Press, New York, 2003).

[2] L. Mandel and E. Wolf, Optical Coherence and Quantum Optics (Cambridge Univ. Press, Cambridge, 1995).

[3] G. Weihs, T. Jennewein, C. Simon, H. Weinfurter, and A. Zeilinger, "Violation of Bell's inequality under strict Einstein locality conditions," Phys. Rev. Lett. 81, 50395043 (1998).

[4] M. Genovese, "Research on hidden variable theories: A review of recent progresses," Phys. Rep. 413, 319-396 (2005).

[5] D. Bouwmeester, J. W. Pan, K. Mattle, M. Eibl, H. Weinfurter, and A. Zeilinger, "Experimental quantum teleportation," Nature 390, 575-579 (1997).

[6] M. Genovese, "Real applications of quantum imaging," J. Opt. 18, 073002 (2016).

[7] O. Jedrkiewicz, Y. K. Jiang, E. Brambilla, A. Gatti, M. Bache, L. A. Lugiato, and P. Di Trapani, "Detection of sub-shot-noise spatial correlation in high-gain parametric down-conversion," Phys. Rev. Lett. 93, 243601 (2004).

[8] O. Haderka, J. Peřina Jr., M. Hamar, and J. Peřina, "Direct measurement and reconstruction of nonclassical features of twin beams generated in spontaneous parametric down-conversion," Phys. Rev. A 71, 033815 (2005).

[9] M. Bondani, A. Allevi, G. Zambra, M. G. A. Paris, and A. Andreoni, "Sub-shot-noise photon-number correlation in a mesoscopic twin beam of light," Phys. Rev. A 76, 013833 (2007).

[10] J.-L. Blanchet, F. Devaux, L. Furfaro, and E. Lantz, "Measurement of sub-shot-noise correlations of spatial fluctuations in the photon-counting regime," Phys. Rev. Lett. 101, 233604 (2008).

[11] G. Brida, L. Caspani, A. Gatti, M. Genovese, A. Meda, and I. R. Berchera, "Measurement of sub-shot-noise spa- tial correlations without backround subtraction," Phys. Rev. Lett. 102, 213602 (2009).

[12] D. N. Klyshko, "Use of two-photon light for absolute calibration of photoelectric detectors," Sov. J. Quantum Electron. 10, 1112 (1980).

[13] G. Brida, M. Genovese, and M. Gramegna, "Twinphoton techniques for photo-detector calibration," Laser Phys. Lett. 3, 115-123 (2006).

[14] J. Peřina Jr., O. Haderka, M. Hamar, and V. Michálek, "Absolute detector calibration using twin beams," Opt. Lett. 37, 2475-2477 (2012).

[15] O. Haderka, J. Peřina Jr., V. Michálek, and M. Hamar, "Absolute spectral calibration of an intensified CCD camera using twin beams," J. Opt. Soc. Am. B 31, B1B7 (2014).

[16] J.G. Rarity and P.R. Tapster, "Quantum interference: experiments and applications," Phil. Trans. R. Soc. A 355, 2267-2277 (1997).

[17] J. Laurat, T. Coudreau, N. Treps, A. Maitre, and C. Fabre, "Conditional preparation of a quantum state in the continuous variable regime: Generation of a subPoissonian state from twin beams," Phys. Rev. Lett. 91, 213601 (2003).

[18] H. Zou, S. Zhai, J. Guo, R. Yang, and J. Gao, "Preparation and measurement of tunable highpower sub-Poissonian light using twin beams," Opt. Lett. 31, 1735-1737 (2006).

[19] J. Peřina Jr., O. Haderka, and V. Michálek, "SubPoissonian-light generation by postselection from twin beams," Opt. Express 21, 19387-19394 (2013).

[20] M. Lamperti, A. Allevi, M. Bondani, R. Machulka, V. Michálek, O. Haderka, and J. Peřina Jr., "Optimal sub-Poissonian light generation from twin beams by photon-number resolving detectors," J. Opt. Soc. Am. B 31, 20-25 (2014). 
[21] T. S. Iskhakov, V. C. Usenko, U. L. Andersen, R. Filip, M. V. Chekhova, and G. Leuchs, "Heralded source of bright multi-mode mesoscopic sub-Poissonian light," Opt. Lett. 41, 2149-2152 (2016).

[22] G. Harder, T. J. Bartley, A. E. Lita, S. W. Nam, T. Gerrits, and C. Silberhorn, "Single-mode parametric-downconversion states with 50 photons as a source for mesoscopic quantum optics," Phys. Rev. Lett. 116, 143601 (2016).

[23] B. Y. Zeldovich and D. N. Klyshko, "Field statistics in parametric luminescence," Sov. J. Exp. Theor. Phys. Lett. 9, 40 (1969).

[24] J. Peřina Jr., O. Haderka, and J. Soubusta, "Quantum cryptography using a photon source based on postselection from entangled two-photon states," Phys. Rev. A 64, 052305 (2001).

[25] O. Alibart, D. B. Ostrowsky, P. Baldi, and S. Tanzilli, "High-performance guided-wave asynchronous heralded single-photon source," Opt. Lett. 30, 1539-1541 (2008).

[26] G. Brida, I. P. Degiovanni, M. Genovese, F. Piacentini, P. Traina, A. Della Frera, A. Tosi, A. Bahgat Shehata, C. Scarcella, A. Gulinatti, M. Ghioni, S. V. Polyakov, A. Migdall, and A. Giudice, "An extremely low-noise heralded single-photon source: A breakthrough for quantum technologies," Appl. Phys. Lett. 101, 221112 (2012).

[27] D. B. Horoshko, S. De Bievre, G. Patera, and M. I. Kolobov, "Thermal-difference states of light: Quantum states of heralded photons," Phys. Rev. A 100, 053831 (2019).

[28] B. E. A. Saleh and M. C. Teich, "Can the channel capacity of a light-wave communication system be increased by the use of photon-number-squeezed light?" Phys. Rev. Lett. 58, 2656-2659 (1987).

[29] M. A. Nielsen and I. L. Chuang, Quantum Computation and Quantum Information (Cambridge Univ. Press, Cambridge, 2000).

[30] E. Jakeman and J. G. Rarity, "The use of pair production processes to reduce quantum noise in transmission measurements," Opt. Commun. 59, 219-223 (1986).

[31] G. Brida, M. Genovese, and I. R. Berchera, "Experimental realization of sub-shot-noise quantum imaging," Nat. Photon. 4, 227-230 (2010).

[32] R. Whittaker, C. Erven, A. Neville, M. Berry, J. L. OBrien, H. Cable, and J. C. F. Matthews, "Absorption spectroscopy at the ultimate quantum limit from singlephoton states," New J. Phys. 19, 023013 (2017).

[33] M. Li, C.-L. Zou, D. Liu, G.-P. Guo, G.-C. Guo, and X.-F. Ren, "Enhanced absorption microscopy with correlated photon pairs," Phys. Rev. A 98, 012121 (2018).

[34] J. Sabines-Chesterkind, A. R. McMillan, P. A. Moreau, S. K. Josh, S. Knauer, E. Johnston, J. G. Rarity, and J. C. F. Matthews, "Twin-beam sub-shot-noise rasterscanning microscope," Opt. Express 27, 30810 - 30818 (2019).

[35] A. F. Abouraddy, K. C. Toussaint Jr., A. V. Sergienko, B. E. A. Saleh, and M. C. Teich, "Entangled-photon ellipsometry," J. Opt. Soc. Am. B 19, 656-662 (2002).

[36] V. Giovannetti, S. Lloyd, and L. Maccone, "Quantum metrology," Phys. Rev. Lett. 96, 010401 (2006).

[37] V. Giovannetti, S. Lloyd, and L. Maccone, "Advances in quantum metrology," Nat. Photon. 5, 222-229 (2011).

[38] G. S. Agarwal and K. Tara, "Nonclassical character of states exhibiting no squeezing or sub-Poissonian statistics," Phys. Rev. A 46, 485-488 (1992).
[39] T. S. Iskhakov, V. C. Usenko, R. Filip, M. V. Chekhova, and G. Leuchs, "Low-noise macroscopic twin beams," Phys. Rev. A 93, 043849 (2016).

[40] S. M. Barnett, G. Ferenczi, C. R. Gilson, and F. C. Speirits, "Statistics of photon subtracted and photon-added states," Phys. Rev. A 98, 013809 (2018).

[41] M. S. Kim, E. Park, P. L. Knight, and H. Jeong, "Nonclassicality of a photon-subtracted gaussian field," Phys. Rev. A 71, 043805 (2005).

[42] O. S. Magańa-Loaiza, R. de J. León-Montiel, A. PerezLeija, A. B. URen, C. You, K. Busch, A. E. Lita, S. W. Nam, R. P. Mirin, and T. Gerrits, "Multiphoton quantum-state engineering using conditional measurements," npj Quant. Inf. 5, 80 (2019).

[43] C. K. Hong and L. Mandel, "Experimental realization of a localized one-photon state," Phys. Rev. Lett. 56, 58 60 (1986).

[44] J. Peřina Jr., M. Centini, C. Sibilia, M. Bertolotti, and M. Scalora, "Anti-symmetric entangled two-photon states generated in nonlinear GaN/AlN photonic-bandgap structures," Phys. Rev. A 75, 013805 (2007).

[45] W. A. T. Nogueira, S. P. Walborn, S. Padua, and C. H. Monken, "Experimental observation of spatial antibunching of photons," Phys. Rev. Lett. 86, 4009-4012 (2001).

[46] D. P. Caetano and P. H. Souto Ribeiro, "Generation of spatial anti-bunching with free-propagating twin beams," Phys. Rev. A 68, 043806 (2003).

[47] M. Zukowski, A. Zeilinger, M. A. Horne, and A. K. Ekert, "'Event-ready-detectors" Bell experiment via entanglement swapping," Phys. Rev. Lett. 71, 4287-4290 (1993).

[48] A. Scherer, R. B. Howard, B. C. Sanders, and W. Tittel, "Quantum states prepared by realistic entanglement swapping," Phys. Rev. A 80, 062310 (2009).

[49] L. M. Duan, M. D. Lukin, J. I. Cirac, and P. Zoller, "Long-distance quantum communication with atomic ensembles and linear optics," Nature 414, 413-419 (2001).

[50] C. W. Chou, H. de Riedmatten, D. Felinto, S. V. Polyakov, S. J. van Enk, and H. J. Kimble, "Measurement-induced entanglement for excitation stored in remote atomic ensembles," Nature 438, 828832 (2005).

[51] B. E. A. Saleh, Photoelectron Statistics (Springer-Verlag, New York, 1978).

[52] J. Peřina Jr., V. Michálek, and O. Haderka, "Nonclassicality of optical fields as observed in photocount and photon-number distributions," Opt. Express 28, 3262032631 (2020).

[53] I. I. Arkhipov, J. Peřina Jr., V. Michálek, and O. Haderka, "Experimental detection of nonclassicality of single-mode fields via intensity moments," Opt. Express 24, 29496-29505 (2016).

[54] C. T. Lee, "Measure of the nonclassicality of nonclassical states," Phys. Rev. A 44, R2775-R2778 (1991).

[55] J. Peřina, Quantum Statistics of Linear and Nonlinear Optical Phenomena (Kluwer, Dordrecht, 1991).

[56] J. Peřina Jr., I. I. Arkhipov, V. Michálek, and O. Haderka, "Non-classicality and entanglement criteria for bipartite optical fields characterized by quadratic detectors," Phys. Rev. A 96, 043845 (2017).

[57] J. Peřina Jr., O. Haderka, and V. Michálek, "Nonclassicality and entanglement criteria for bipartite optical fields characterized by quadratic detectors II: Crite- 
ria based on probabilities," Phys. Rev. A 102, 043713 (2020).

[58] R. J. Glauber, "Coherent and incoherent states of the radiation field," Phys. Rev. 131, 2766-2788 (1963).

[59] E. C. G. Sudarshan, "Equivalence of semiclassical and quantum mechanical descriptions of statistical light beams," Phys. Rev. Lett. 10, 277-179 (1963).

[60] I. S. Gradshtein and I. M. Ryzhik, Table of Integrals, Series, and Products, 6th ed. (Academic Press, San Diego, 2000).

[61] J. Peřina Jr., M. Hamar, V. Michálek, and O. Haderka, "Photon-number distributions of twin beams generated in spontaneous parametric down-conversion and measured by an intensified CCD camera," Phys. Rev. A $\mathbf{8 5}$, 023816 (2012).

[62] J. Peřina Jr., O. Haderka, V. Michálek, and M. Hamar, "State reconstruction of a multimode twin beam using photodetection," Phys. Rev. A 87, 022108 (2013).

[63] A. P. Dempster, N. M. Laird, and D. B. Rubin, "Maximum likelihood from incomplete data via the EM algorithm," J. Royal Statist. Soc. B 39, 1-38 (1977).

[64] Y. Vardi and D. Lee, "From image deblurring to optimal investments: Maximum likelihood solutions for positive linear inverse problems," J. Royal Statist. Soc. B 55,
$569-612(1993)$.

[65] W. Vogel, "Nonclassical correlation properties of radiation fields," Phys. Rev. Lett. 100, 013605 (2008).

[66] A. Miranowicz, M. Bartkowiak, X. Wang, Y.-X. Liu, and F. Nori, "Testing nonclassicality in multimode fields: A unified derivation of classical inequalities," Phys. Rev. A 82, 013824 (2010).

[67] D. N. Klyshko, "Observable signs of nonclassical light," Phys. Lett. A 213, 7-15 (1996).

[68] E. Waks, E. Diamanti, B. C. Sanders, S. D. Bartlett, and Y. Yamamoto, "Direct observation of nonclassical photon statistics in parametric down-conversion," Phys. Rev. Lett. 92, 113602 (2004).

[69] E. Waks, B. C. Sanders, E. Diamanti, and Y. Yamamoto, "Highly nonclassical photon statistics in parametric down-conversion," Phys. Rev. A 73, 033814 (2006).

[70] K. Wakui, Y. Eto, H. Benichi, S. Izumi, T. Yanagida, K. Ema, T. Numata, D. Fukuda, M. Takeoka, and M. Sasaki, "Ultrabroadband direct detection of nonclassical photon statistics at telecom wavelength," Sci. Rep. 4, 4535 (2014).

[71] P. M. Morse and H. Feshbach, Methods of Theoretical Physics, Vol. 1 (McGraw-Hill, Amsterdam, 1953). 